Tiіu Reimo

\title{
TOD! - GRAB! - VERWESUNG! VISUALISIERUNG VON TOD UND STERBEN IN DEN ESTNISCHEN FREMDSPRACHIGEN FUNERALDRUCKEN DES 17. UND 18. JAHRHUNDERTS
}

Tod! - Grab! - Verwesung! - welcher schreckliche Gedanke! - Sterben; dieses Leben verlieren, welches so tausendfach uns reisset - aufhören, mit diesem Auge die Schönheit der Natur zu sehen, mit diesem Ohr entzückende Gesänge zu hören, mit dieser Brust Wonne zu athmen - Freunde verlassen, die in Thränen schmelzen - ohnmächtig ins Grab sinken - den Körper der Verwesung und den Würmern zum Raube zu übergeben - ganz vernichtet $z u$ werden oder in einen Zustand zu kommen, den wir nicht kennen, vor dem die Natur erzittert - grausame Gedanken! - Tod! Was bist du für ein fürchterlicher Auftritt unter den Menschen?

Ernst Wilhelm August Hörschelmann, Professor des Tallinner Gymnasiums, beginnt mit diesen Worten den Nachruf an

DOI: https://doi.org/10.12697/BJAH.2019.17.01

Der Aufsatz wurde im Rahmen des Projektes EKKM 14-330 „Die Zusammenstellung und Redaktion der estnischen fremdsprachigen retrospektiven Bibliographie“ angefertigt. Die Übersetzung aus dem Estnisch stammt von Helje-Laine Kannik, die Abbildungen wurden von Rene Haljasmäe digitalisiert. 
Adrian Heinrich Frese, den im Jahre 1779 verstorbenen Tallinner Bürgermeister und Syndikus. ${ }^{1}$

Jahrhunderte lang war der Tod aufgrund von Kriegen, Hungersnöten, der Pest und anderen Epidemien der ständige Begleiter der Lebenden. Es bildeten sich im Laufe der Zeit Bestattungsbräuche heraus sowie auch Arten und Weisen, wie man über den Tod spricht, wie man Trostworte für die Hinterbliebenen findet, wie man den Verstorbenen verewigt - das heißt, wie man den Tod kommuniziert. So sind auch in heutigen Bestattungsbräuchen alte rituelle Sitten sowie mit dem Tod und der Trauer verbundene Symbole (ikonische Zeichen) klar erkennbar.

Ars moriendi - die Kunst des Sterbens - hat in Estland im letzten Jahrzehnt interessantes Forschungsmaterial vor allem für Kulturund Kunsthistoriker geboten. Erinnern wir uns an die am Seelentag im Jahre 2012 im Museum der Tallinner St. Nikolaikirche eröffnete gleichnamige Ausstellung ${ }^{2}$, die Abhandlung über Beerdigungsbräuche in Tallinn von Sulev Mäeväli ${ }^{3}$, die Untersuchung zur mittelalterlichen Sterbekultur von Mari Loit ${ }^{4}$ oder den in den Veröffentlichungen des Estnischen Nationalarchivs erschienenen Artikel „Trost und Gedenken“ von Krista Kodres, in dem die Autorin aufzeigt, dass auch für die evangelische Kirche Bilder nützliche Mittel beim Erlernen und Erinnern des Gotteswortes gewesen sind. ${ }^{5}$

In der den Verstorbenen bzw. die Verstorbene darstellenden Kunst wurde neben der Verewigung bisher wenig Aufmerksamkeit auf die mit den Bestattungen verbundenen Druckschriften gerichtet -

1 Ernst August Wilhelm Hörschelmann, Standrede bey der Beerdigung des Hochedelgebohrnen und Hochgelahrten Herrn Adrian Heinrich Frese, der Kaiserlichen Stadt Reval Hochverdienten

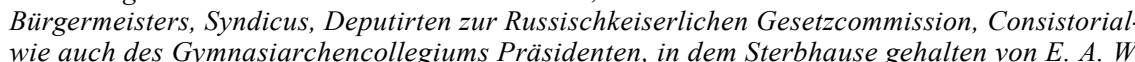
wie auch des Gymnasiarchencollegiums Präsidenten, in dem Sterbhause gehalten von E. A. W. Hoerschelmann, der Philosophie Pr Universität Tallinn [Tallinna Ülikooli Akadeemiline Raamatukogu,

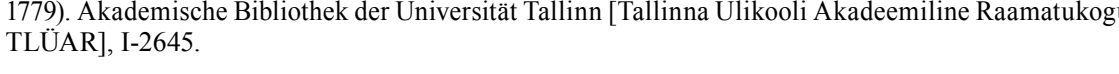
2 Ars moriendi - suremise kunst: näitus Niguliste muuseumis 02.11.2012-02.06.2013 $=$ Ars Moriendi - the Art of Dying: Exhibition in the Niguliste Museum 02.11.2012-02.06.2013 (Tallinn: Eesti Kunstimuuseum, 2013).

3 Sulev Mäeväli, ,,Mõnda matusekommetest Tallinnas 17.-19. sajandil“, Tallinna Linnamuuseum aastaraamat 1996/1997 (Tallinn: Teaduste Akadeemia Kirjastus, 1997), 126-144.

4 Mari Loit, „Keskaegsest surmakultuurist ja hauatähistest reformatsioonieelse Tallinna kirikutes ja kloostrites“, Vana Tallinn, 17 (21) (2006), 13-190.

5 Krista Kodres, „Trööst ja mäle(s)tamine: matuserituaal ja memoriaalkunst varauusaegse Eestis“, Kroonikast epitaafini. Eesti- ja Liivimaa varauusaegsest kultuurielust (Tartu Rahvusarhiiv, 2017), 436-465, hier 457.
Predigten und Trauergedichtsammlungen, die ebenfalls mit dem Tod Verbundenes sowohl in Text als auch in Bildern vermitteln. Im Folgenden habe ich das Ziel, einen Überblick über die Visualisierung von Tod und Sterben in Estland erschienenen fremdsprachigen Druckschriften des 17. und 18. Jahrhunderts zu geben.

Die Idee für den Artikel entstand im Laufe der Zusammenstellung der retrospektiven Bibliografie der estnischen fremdsprachigen Druckschriften. Ende des Jahres 2016 fingen wir an, die Verzierungselemente der Druckschriften des 17. und 18. Jahrhunderts zu scannen, um eine Auswahl für die Illustrationen der geplanten Druckausgabe der Bibliografie zu schaffen. Von den uns bekannten Drucken des 17. und 18. Jahrhunderts bilden die Leichenpredigten und Trauergedichtsammlungen einen bemerkenswerten Teil, von denen die meisten im 17. Jahrhundert erschienen sind. ${ }^{6}$ Während ich das Erklären der Komposition und der Botschaft der Leichenpredigten den Theologen und die Analyse der Trauerdichtung den Literaturwissenschaftlern überlasse, konzentriere ich mich im vorliegenden Artikel auf die Ornamentik der Druckschriften. Die die Funeraldrucke schmückenden Vignetten, Zierleisten, Zierinitialen und Zierrahmen werden gewöhnlich nicht zur Buchillustration gezählt, jedoch geben sie dem Text zusätzlich eine starke visuelle Bedeutung. Die wenigen Untersuchungen zur estnischen frühneuzeitlichen Buchgestaltung sind in der ersten Hälfte des 20. Jahrhunderts veröffentlicht worden; es sind nur zwei bekannt und beide behandeln das Buchgestaltung des estnischsprachigen Buches. ${ }^{7}$

Im vorliegenden Artikel konzentriere ich mich wegen des großen Umfangs des Materials auf die in den Drucken verwendeten Vignetten. Das hier Dargestellte ist nicht vollständig, die Verzeichnung der verschiedenen dekorativen Elemente wird fortgesetzt, jedoch ist es bereits möglich, eine allgemeine Vorstellung von den in den Funeraldrucken auftretenden Vignetten zu bekommen. Die Leichenpredigten und Trauergedichte beinhaltenden Drucke gehören zur Gelegenheitsliteratur - sie wurden in Bezug auf ein

6 Ein Überblick über die geistlichen Druckschriften der Schwedenzeit erschien im vorigen Jahr in den Veröffentlichungen des Estnischen Staatsarchivs. Tiiu Reimo, Helje-Laine Kannik, Tallinna rootsiaegsed vaimuliku sisuga trükised“, Kroonikast epitaafini. Eesti- ja Liivima varaunsaegsest kultuurielust (Tartu: Rahvusarhiiv, 2017), 270-321.

7 Hugo Peets, ,Die graphische Ausstattung der estnischen Drucke des 17. und 18. Jahrhunderts“; Gutenberg-Jahrbuch (Mainz, 1937), 217-227; Veronika Sikk, Eesti raamatu välimusest XVII ja XVIII sajandil (Tartu: Teaduslik Kirjandus, 1941). 
bestimmtes Ereignis und eine konkrete Person geschrieben. In der deutschen Literatur werden solche Drucke auch Personalschriften genannt, was die Besonderheit dieser Drucke sogar besser zum Ausdruck bringt. Den den Artikel illustrierenden Bildern wurden die Maße des Originals in Millimetern hinzugefügt; den Ort der Druckerei bezeichnen die Namen der Städte: TALLINN, TARTU, NARVA und PÄRNU. Als Vergleichsmaterial werden finnische und schwedische frühneuzeitliche Drucke benutzt. Der Vergleich mit Vignetten, die in finnischen Druckereien verwendet wurden, wird ermöglicht durch den von Anna Peräla herausgegebenen zweiteiligen „Typographischen Atlas Finnlands“, in dem alle bis zum Jahre 1827 in finnischen Druckereien verwendeten Lettern, Zierleisten und Vignetten zusammengestellt sind. ${ }^{8}$ Gutes Vergleichsmaterial erhalten wir auch in der Bibliografie „Deutschsprachige Gelegenheitsdichtung in Stockholm und Uppsala zwischen 1613 und 1719“ von Jan Drees. Neben der Beschreibung der Gelegenheitsdrucke beinhaltet sie auch Beispiele zu den sich in den Gelegenheitsdrucken befindenden Vignetten. ${ }^{9}$

\section{DIE ERINNERUNG AN DEN VERSTORBENEN BEWAHREN}

In der Frühen Neuzeit wurden die Bestattungsrituale in Estland durch Kirchenordnungen und das Landrecht geregelt, durch welche die Ordnung der kirchlichen Handlung, der Begräbnisstätte, des Leichenzuges, des Sargbeschlags und des Leichenschmauses festgelegt wurden. Die Funeraldruckschriften regulierenden Verordnungen oder Regelungen haben wir bisher nicht gefunden. Die Angaben der uns bekannten Druckschriften erlauben es, diese in zwei große Gruppen zu teilen: Leichenpredigten und Trauergedichte. Die bis heute erhaltenen Drucke geben uns keine vollständigen Informationen über alles im Druck Erschienene, jedoch ermöglichen sie es, eine Vorstellung von der Struktur und der typographischen Gestaltung zu bekommen.

8 Anna Perälä, Suomen typografinen atlas $=$ Finsk typografisk atlas $=$ Typographischer Atlas Finnlands: 1642-1827, I-II (Helsinki: Helsinkin Yliopiston Kirjasto, 2000).

9 Jan Drees, Deutschsprachige Gelegenheitsdichtung in Stockholm und Uppsala zwischen 1613-1719. Bibliographie der Drucke nebst einem Inventar der in ihnen verwendeten dekorativen Druckstöcke (Stockholm: Kungl. Biblioteket, 1995).
Das schwedische Kirchengesetz aus dem Jahre 1686, mit dem die Kirchenordnung im ganzen schwedischen Reich - darunter in Est- und Livland sowie auf Ösel - vereinheitlicht wurde, mahnt Begräbnisfeiern zurückhaltend durchzuführen:

Das Volck soll auch gemahnet werden, bey Leichbegängnüssen und Begräbnüssen, sich nach Unsern Verordnungen und Satzungen zu richten und nachmässig zu bezeigen, bevorab, dass sie von kostbahren Sargen und Ankleidung der Todten ablassen; Immassen hinfüro nur etzliche wenige der nechsten Anverwandten und keine andere dazu sollen gebrauchet und geladen werden. ${ }^{10}$

Im Gesetz ist auch die Zeit der Leichenpredigt genau festgelegt worden: wenn der Verstorbene auf dem Kirchhof beerdigt wird, dann hört man die Predigt nachdem der Sarg in das Grab gesenkt wurde; wird der Verstorbene in der Kirche begraben, hört man die Predigt bevor der Sarg in das Grab gesenkt wird:

Wann die Leiche auff dem Kirchhoffe begraben wird, muss sie nicht erst in die Kirche getragen werden, ... sondern alssbald niedergesetzet und in die Erde gesäncket werden; Darauff geht man in die Kirche, umb die Predigt $z u$ hören. Wird die Leiche in der Kirchen begraben, so soll sie mitten vor die Cantzel gesetzet und nach gehaltener Predigt ins Grab gesencket werden.

Die Personalien sollen nach eines jedweden Leben eingerichtet und ohne Weitläuftigkeit samt unverdientem und vergeblichem Ruhm abgefasset werden. ${ }^{11}$

Die Leichenpredigten können ziemlich umfangreich sein und in den meisten Fällen beinhalten sie auch Angaben über die Herkunft des bzw. der Verstorbenen und seinen bzw. ihren Lebenslauf. Im Druck erschienen die Leichenpredigten von Angehörigen der sozialen Oberschichten: Adlige, hohe Staatsbeamte, Militärleute, Geistliche, Universitäts- und Gymnasialprofessoren, Ratsherren und deren Familienmitglieder. In Estland wurden Leichenpredigten und Trauerpoesie auch aus Anlass des Todes der Herrscher - der schwedischen Könige sowie russischen Zaren - herausgegeben, 10 Kirchen-Gesetz und Ordnung, So der Grossmächtigste König und Herr, Herr Carl der Eilffte,
Der Schweden, Gothen und Wenden König Im Jahr 1686. hat verfassen und Im Jahr 1687. im Druck aussgehen und publiciren lassen. Mit denen dazu gehörigen Verordnungen. Auff HöchstErmeldten Ihrer Königl. Majest. gnädigsten Befehl ins Teutsche übersetzet (Riga: Johann Georg Wilcken, 1687), 91-92, Cap. XVIII. Von Christlichen Begräbnis, \$2.

11 Ibidem ,93-94, Cap. XVIII, §6-\$7. 
obwohl eine direkte Verbindung zu ihnen fehlte. ${ }^{12}$ Die Funeraldrucke waren von der Gestaltung her zurückhaltend: zur Verschönerung ein Zierrahmen auf dem Titelblatt, Anfangsleisten und Zierinitialen, sehr selten eine Vignette am Anfang oder am Ende des Textes.

Es war üblich, schon auf dem Titelblatt möglichst viele Angaben zu dem Verstorbenen bzw. der Verstorbenen anzuführen: be Staatsbeamten wurden alle Positionen angegeben, bei Militärsleuten die Todesumstände, beim Tode des Ehemannes oder der Ehefrau wurden Liebe und Treue betont. Bei vielen Funeraldrucken findet sich auf dem Titelblatt der Vermerk, dass der Druck dieser Trauerschrift auf Wunsch der Angehörigen geschehen ist (auff Begehren zum Druck abgefertiget; auff der hochbetrübten Fraw Witwen Begehren im Druck herfür gegeben). Die Leichenpredigt ergänzten dem Verstorbenen gewidmete und den Trauernden zum Trost geschriebene Versausgaben, die als Anhang des Predigttextes oder als eigenständige Drucke erschienen. Wie viel solche Drucke kosteten und wer die Kosten für die von Universitäts- und Gymnasialprofessoren oder städtischen Geistlichen geschriebenen Gedichte trug, ist nicht bekannt. In der Beschreibung der Begräbniskosten des Freiherrn Konrad von Uexküll-Güldenband ist aufgezeichnet, dass für den Druck des Gesangblatts und des in memoriam 20 Reichstaler bezahlt wurden; das Drucken der Predigt von Magister Justus Heinrich Oldekop in der Tallinner Domkirche kostete 36 Reichstaler und für ein Gedicht zum Andenken an Herrn Uexküll wurden dem Professor Müller 25 Reichstaler gegeben..$^{13}$

Größere Aufmerksamkeit auf die Visualisierung des Todes wurde in Trauergedichten gerichtet, die Berufskollegen oder Verwandte herausgegeben haben. Größtenteils sind diese Drucke von geringem Umfang, zwei- bis vierseitige Trostzeilen eines Autors oder auch

12 Z. B. wurde 1634 in Tartu eine Ode von Timotheus Polus zum Lobpreis an Gustav Adolf publiziert: Gustavus Adolphus Victor Magnus. Oder Die Erlösete Kirche,vnd Teutsche Freyheit. Durch Rahten vnd Thaten, vnd durch Muth vnd Blut des Glorwürdigsten Heldes vnd König. Gustavi Adolphi Magni Ritterlich erhalten. Historisch vnd Poetisch zusammen getragen vnd genommen theils aus Torquato Tasso, theils auss Opitio \&c. Durch Timotheum Polum, P.L. (Dorpat: Jacob Becker, 1634); Im Jahre 1725 wurde in Tallinn der Text einer Kantate, die am 2. Bey Der Am 2ten Junii des 1725ten Jahres in Reval gehaltenen Gedächtniss-Predigt über Das Hochseelige Absterben Ihro Käyserl. Majest. Petri I des Grosen, SelbstHaltern des gantzen Reusslandes, und Vatern des Vaterlandes etc. etc. etc. Glorwïrdigsten Andenckens wolte seine unterthänigste Pflicht abstatten in folgender Cantata, und in allertiefster De- und Weh-muht absingend bezeugen Christian Ludwig Heimbrod Käyserl. Postmeister zu Reval (Reval: Johan Köhler, 1725).

13 Mäeväli, ,Mõnda matusekommetest Tallinnas 17.-19. sajandil“, 128-129. Die Leichenpredig von Oldekop ist nicht erhalten geblieben.

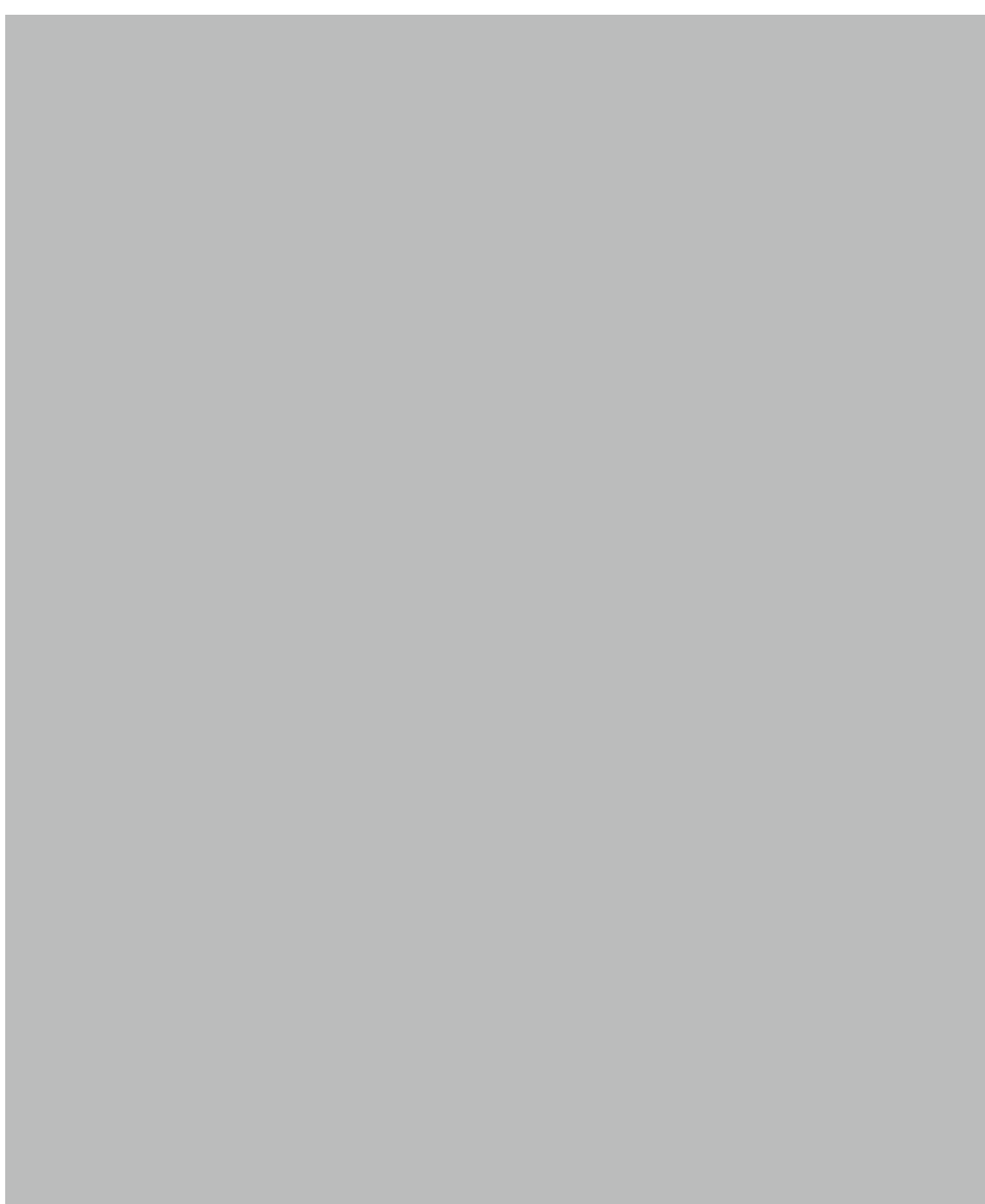

Abb. 1. Ein typisches Titelblatt einer Druckausgabe der Leichenpredigt im 17. Jahrhundert: Zwei Predigten von Ericus von Beeck anlässlich des Todes von Philipp von Scheiding (1578-1646), Gouverneur von Estland, bei der Verabschiedung am 7. Juli 1646 und beim Begräbnis am 16. Februar 1647 in der St. Olaikirche. Gedruckt in Reval bei Heinrich Westphal im Jahre 1647. (Es wurde diese Leichenpredigt und dazu einige Trauergedichte gedruckt, heute sind vier davon uns bekannt und sie werden in der Universitätsbibliothe
Uppsala aufbewahrt.)

Gedichtsammlungen mit Beileidsbekundung mehrerer Verfasser. Es gibt auch umfangreiche Gedichthefte, die Gedichte in verschiedenen Sprachen (Latein, Deutsch, Griechisch, Estnisch) enthalten: unter 
jedem Gedicht steht der Name und das Amt des Verfassers. In vielen Fällen zogen die Autoren es vor, anonym zu bleiben und nannten sich einen guten Freund oder untertänigen Diener; oder fügten nur die Initialen des Namens hinzu, die es heute nicht in allen Fällen gelingt zu entschlüsseln.

Die Gedichthefte der Schwedenzeit sind meist in Quartformat, in der ersten Hälfte des 18. Jahrhunderts sind sie überwiegend in Folioformat und am Ende des 18. Jahrhunderts wieder in Quart- oder Oktavformat. Wegen des kleinen Umfangs fallen die typografischen dekorativen Elemente mehr auf und derer gibt es reichlich. Während die in den Trauerschriften vorkommenden Zierleisten und Zierinitialen die gleichen sind wie in thematisch anderen Texten, tragen vor allem die die Trauerschriften verzierenden Vignetten eine ikonografische Bedeutung; diese lenken die Gedanken der Hinterbliebenen, d. h. der Lebenden, sowohl auf das Mysterium des Todes als auch auf die Symbole des ewigen Lebens.

Die Symbole des Todes in den in Estland erschienenen fremdsprachigen Druckschriften unterscheiden sich nicht von denen, die anderswo in der europäischen christlichen Kultur verwendet wurden, jedoch haben sie bedingt durch die Drucktechnik eine allgemeinere Bedeutung als die in der Monumentalkunst und in der bildenden Kunst bevorzugten Motive. Während die Grabdenkmäler und Epitaphe mit einer konkreten Person verbunden sind, visualisieren die in den Drucken verwendeten Vignetten das mit dem Tod Verbundene sehr allgemein und eignen sich dadurch zum Gedenken aller Menschen unabhängig des Berufs und Alters der Verstorbenen. In den Drucken findet sich auch kein TotentanzMotiv, relativ selten ist die Darstellung des liegenden, sogenannten "schlafenden Verstorbenen ", ${ }^{14}$ es wurden auch weder Wappenschilde, Hausmarken noch Symbole der Evangelisten verwendet. ${ }^{15}$

Von den Symbolen treten Darstellungen von Totenschädel und Sarg in den Vordergrund, die Vergänglichkeit des menschlichen Lebens symbolisieren Sanduhr und Sense. In der zweiten Hälfte des 18. Jahrhunderts kommen auf den Vignetten trauernde Putti und verschiedene Urnenmotive im Empirestil hinzu. Im 17. Jahrhundert

14 Kodres, „Trööst ja mäle(s)tamine: matuserituaal ja memoriaalkunst varauusaegses Eestis“, $453-455$.

15 Loit, „Keskaegsest surmakultuurist ja hauatähistest“, 105-112.

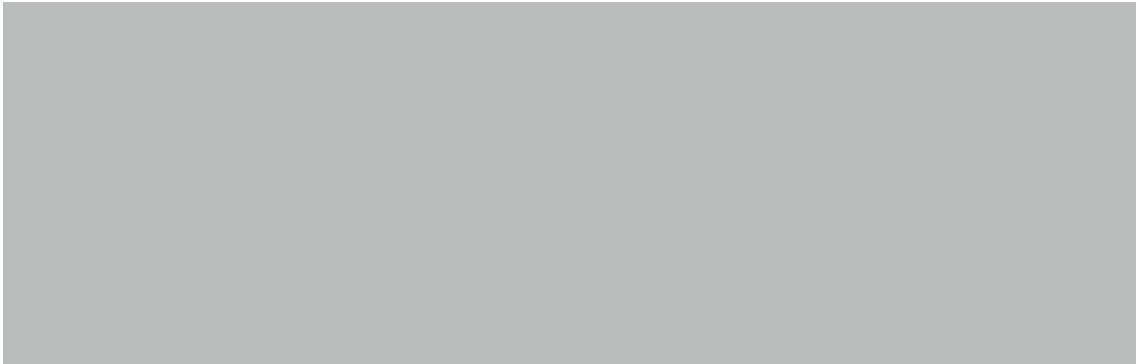

Abb. 2. TALLINN: Ausmass des Originals $20 \times 75$ mm. Eberhard von Renteln, Tröstliche Sterbkunst der Gläubigen Kinder Gottes, Das ist: Eine Christliche Leich- und Gedächtnuss Predigt ... Bey Volckreicher Leichbegängnuss, Des weyland Ehrwürdigen, Achtbahren und Wolgelahrten Herrn M. Ludovici Dunten ... (Reval: Heinrich Westphal, 1640), TLÜAR, I-4221.

wurden der grafischen Gestaltung oft auch Mottos wie „Hodie mihi cras tibi!", "Christus ist mein Leben“, „Nemo hic excipitur", „Memento mori!" angefügt.

Die frühesten, in Tallinner Trauerdrucken verwendeten Motive hat wahrscheinlich der erste Tallinner Buchdrucker Christoph Reusner (ca. 1575-1637, Buchdrucker in Tallinn 1634-1637) als Druckstöcke aus Stockholm mitgebracht. In den Werken seiner Stockholmer Periode sind in den Jahren 1630-1633 solche in Gebrauch gewesen. ${ }^{16}$

Der früheste bis heute erhaltene Trauergedichtdruck, den Vignetten schmücken, ist die Ode von Timotheus Polus zum Gedenken an den Tallinner Ratsherrn Peter von Sprekelsen (ca. 1562-1635). ${ }^{17}$

Das ist eine der wenigen Darstellungen des sogenannten "schlafenden Verstorbenen" samt dem den Tod symbolisierenden Schädel und dem die Vergänglichkeit des menschlichen Lebens darstellenden Stundenglas. Die als Leiste entworfene Vignette ist später beim Drucken der Texte der Leichenpredigten als Verzierung zu Beginn des Textes verwendet worden (siehe Abb. 2). Von Reusner sind zwei registriert, im Falle des nächsten Buchdruckers Heinrich Westphal (?-1653, Wirkungszeit in Tallinn 1638-1653) wissen wir

16 Drees, Deutschsprachige Gelegenheitsdichtung, 338-339. Drees beschreibt die Figur (I.5) als „trauernde[n] Puto“.

17 Timotheus Polus, Ode Auff des weiland Ehrenvesten, Vorachtbarn vnd Wolweisen Herrn Peter von Sprekelsen, der Kön. Seestadt Revall wolverdienten Rathsverwandten tödlichen Hintritt; Welcher Anno 1635. am ersten Pfingsttage im 73. Jahr seines alters in Gott sanfft entschlieff, vnd Freytages hernach begraben ward (Reval: Christoph Reusner, 1635), TLÜAR, XII-947. 


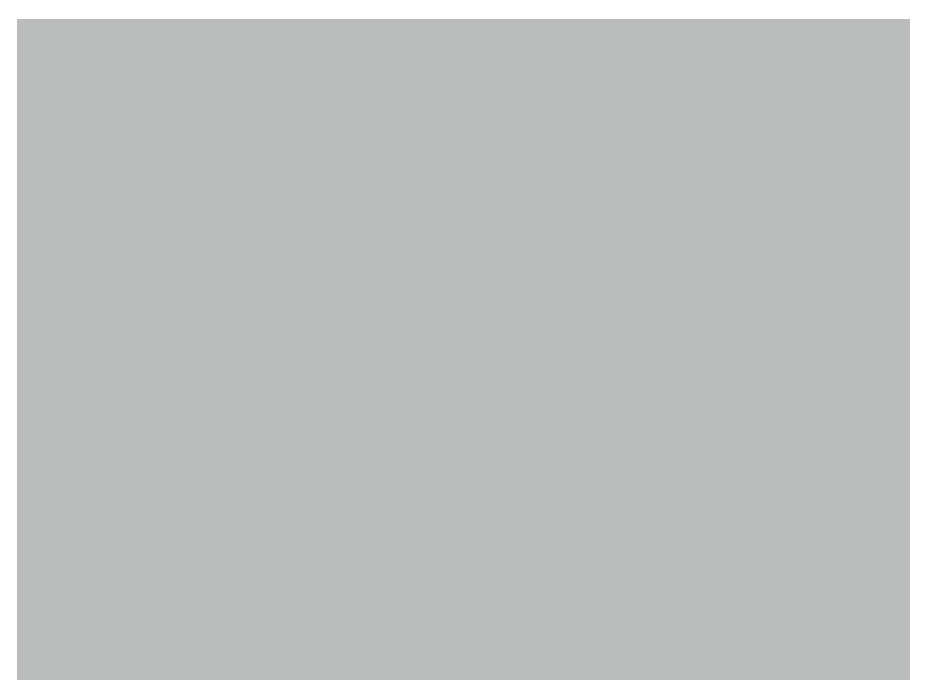

Abb. 3. TARTU: Ausmass des Originals ist nicht bekannt. Ene-Lille Jaanson, Tartu Ülikooli trükikoda 1632-1710 = Druckerei der Universität Dorpat 1632-1710 (Tartu: Tartu Ülikooli Raamatukogu, 2000), 142

von fünf Drucken mit dieser Kopfleiste, der letzte aus dem Jahre 1647. Seit etwa der Mitte des 17. Jahrhunderts kommt die Vignette in Drucken nicht mehr vor.

Die Darstellung des ",schlafenden Verstorbenen“ kommt auch in den Drucken aus der Zeit der Tartuer Academia Gustaviana vor (siehe Abb. 3). ${ }^{18}$

\section{DAS TOTENSCHÄDELMOTIV ALS TODESSYMBOLIK}

Das Beerdigungsgedicht anlässlich des Todes von Spreckelsen schmückt eine andere bei Reusner in Stockholm in Gebrauch gewesene Vignette: das Motiv, zu dem die zwei Jahrhunderte später von Lydia Koidula geschriebenen Gedichtzeilen „meinem Staub die Blum' entblüht" (aus meiner Asche werden Blumen erblühen) oder die Worte aus dem Buch Mose „Im Schweiße deines Angesichts sollst du dein Brot essen, bis du zum Erdboden zurückkehrst, denn ihm bist du entnommen. Denn Erde bist du und zu Erde musst du wieder werden!" (1. Mo 3:19) passen würden. Das Letztgenannte ist

18 Ene-Lille Jaanson, Tartu Ülikooli trükikoda $1632-1710=$ Druckerei der Universität Dorpat 1632-1710 (Tartu: Tartu Ülikooli Raamatukogu, 2000), 142. Die Benutzungszeit der drei Vignetten ist datiert auf die Zeit von 1638 bis 1656, Hinweise auf konkrete Drucke fehlen.
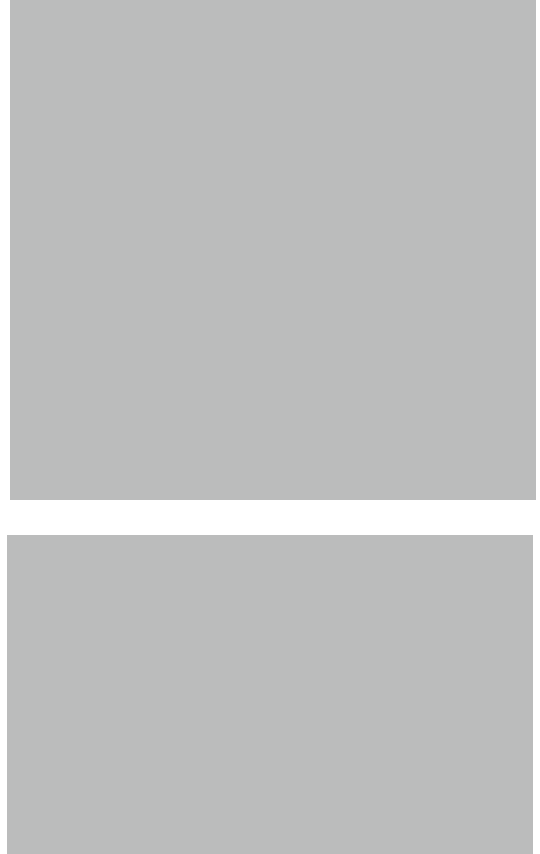
über den Behertzten und beschmertzten Hintritt aus dieser Welt... (Reval: Adolph Simon, 1665).

Abb. 5. TARTU: Ausmass des Originals $35 \times 58 \mathrm{~mm}$. Trauer-Klagen, über die hochklägliche und frühzeitige doch sehlige Todesfälle des Hochwolgebornen Herrn, Hr. Johann-Eberhard von Bellingkhaussen... (Dorpat: Johann Vogel, 1652).

auch heute Teil der Trauerfeier in leicht abgewandelter Form: „Du bist Erde und sollst zu Erde werden."

Das Totenschädelmotiv war im 17. Jahrhundert durchweg unter den Buchdruckern eines der beliebtesten Verzierungselemente der Trauerdrucke, das neben der Vergänglichkeit des menschlichen Lebens auch die Fortsetzung des Lebens symbolisierte. In den estnischen Drucken kommen im Wesentlichen zwei Darstellungen vor: in einem Fall stützt sich der Schädel auf den Boden (siehe Abb. 4), im anderen liegt der Schädel auf den gekreuzten Oberschenkelknochen (siehe Abb. 5). Aus den Augenhöhlen wachsen Halme, Kornähren oder Blumenstängel. In den am Ende des 17. Jahrhunderts erschienenen Narvaer Drucken gibt es noch eine Variante, wo der Totenschädel mit dem gekreuzten Gebein auf dem mit Blumen geschmückten Grab liegt (siehe Abb. 6). 


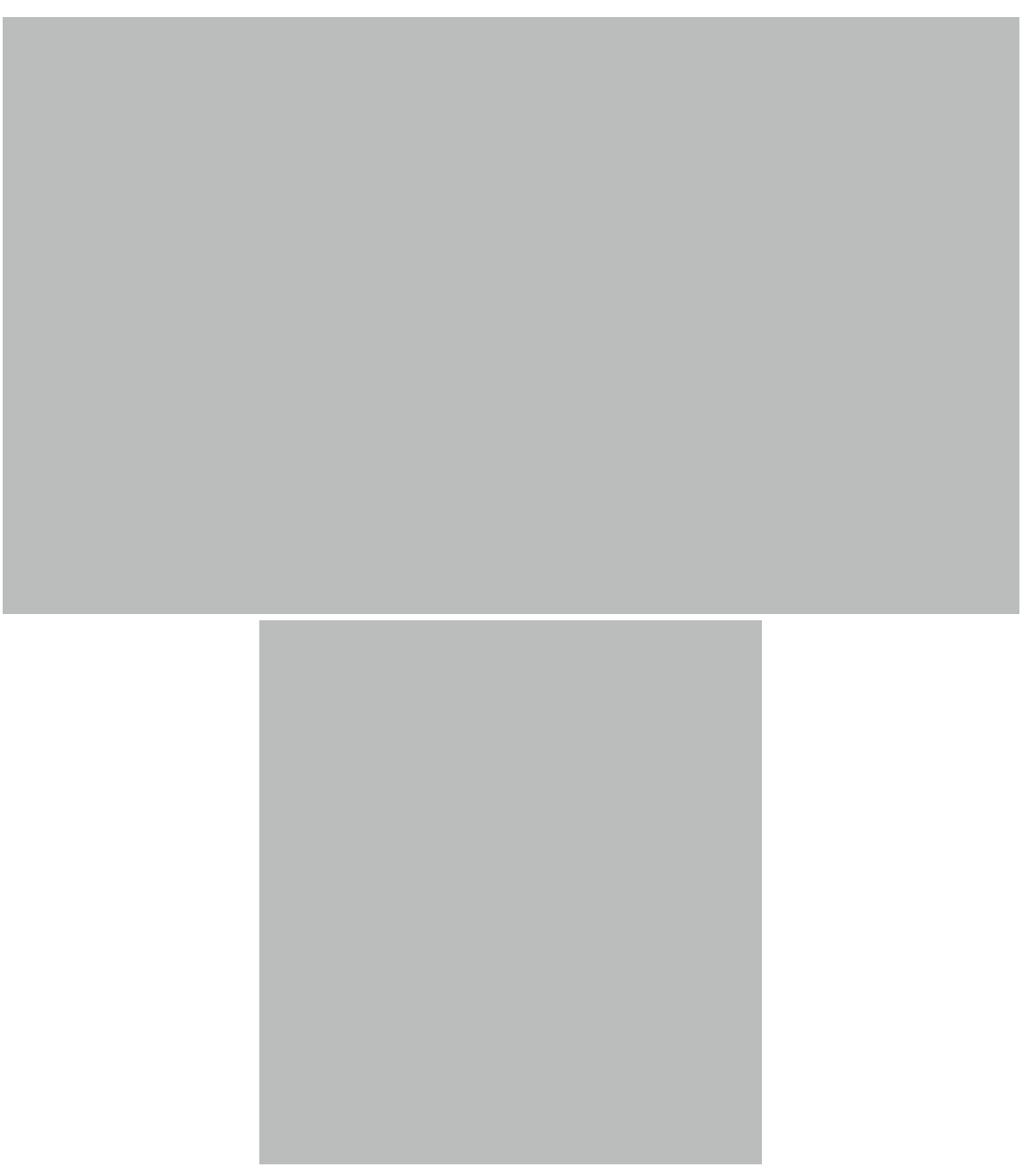

Abb. 6. NARVA: Ausmass des Originals $30 \times 35 \mathrm{~mm}$. Jacob Lang, Gudz Barnas Wandring och Högsta Goda... (Narva: Johann Köhler, 1696).

Abb. 7. TALLINN: Ausmass des Originals $30 \times 30 \mathrm{~mm}$. Der erwählte Leichentext, aus Hesek. Xer fer feylichen Beerdigung des weiland Hochwohlgebohrnen Herrn, Herrn Bernhard Johann von Uxkülls... (Reval: [s.n.], 1761).

Während die Vignette mit der Darstellung des den Todesschlaf schlafenden Verstorbenen hauptsächlich als Kopfleiste am Anfang eines Textes Verwendung gefunden hat, findet man die Totenschädelvignette sowohl als Verzierung des Titelblatts als auch am Anfang oder am Ende eines Textes. Der letzte derzeit registrierte Tallinner Druck mit solche einer Vignette stammt aus dem Jahre
1665..$^{19}$ In Tartu hat der Drucker der Academia Gustaviana Johan Vogel (?-1656, Wirkungszeit in Tartu 1642/1644-1656) eine ähnliche Vignette zur Verzierung von Trauerdrucken benutzt. Bisher ist dies nur von zwei Drucken bekannt, der erste beinhaltet Gedichte anlässlich des Todes der Ehefrau des Landrats Freiherrn Johann Eberhard von Bellingshausen Margaretha von Zöge und seiner Tochter ChristinaElisabeth, die beide in der Rigaer Domkirche begraben wurden; ${ }^{20}$ der zweite Gedichte von 22 Autoren anlässlich des Todes der Ehefrau des Professors der griechischen und der hebräischen Sprache an der Academia Gustaviana Ericus Holstenius Dorothea von Wickeden. ${ }^{21}$

Das Totenschädelmotiv findet sich auch in den am Ende des 17. Jahrhunderts erschienenen Narvaer Drucken. Der aus der Umgebung Rigas gebürtige Johann Köhler (1662-1736, Wirkungszeit in Narva 16951705) wurde 1695 Stadtdrucker in Narva, jedoch erschienen die ersten Druckwerke erst im darauffolgenden Jahr. Nach Angaben Enn Küngs, der die Tätigkeit des Druckers Köhler in Narva basierend auf den sich im Schwedischen Staatsarchiv befindenden Quellen untersucht hat, sind mindestens 30 in Narva gedruckte Werke bekannt. ${ }^{22}$

Von den Beerdigungsdrucken aus Narva sind nur zwei erhalten. Einer darunter ist bekanntlich ist bekanntlich auch der erste in der 19 Traur-Zypressen, über den Behertzten und beschmertzten Hintritt aus dieser Welt, Der VorAchtbahren Hn. Andreas Haeks, WohlVornehmen Bürgers und Handels-Herrn allhie, Weyland hertzgeliebten Ehe-Schatzes, Welche den 20. Septembris itztlauffenden 1665 sten Jahres 29. Jahr ihres Alters in Ihrem Erlöser sanfft und selig entschlaffen und nachgehends den 26. desselben Christüblichen Gebrauch nach in S. Olai Kirchen zur Erden bestätiget wurde. Aus schüldiger Ehren-Pflicht nicht ohne hertz-empfindlichen Beyschmertz Gestecket und vorgestellet Von Stephano Knipern (Reval: Adolph Simon, 1665), TLÜAR, XII-2955.

20 Trauer-Klagen, über die hochklägliche und frühzeitige doch sehlige Todesfälle des Hochwolgebornen Herrn, Hr. Johann-Eberhard von Bellingkhaussen, Freyherrn und Land-Rat des Dörptischen Kräyses in Lifland S. Hertzvielgeliebten Ehgemahls Frauen, Fr. Margarethavon
Zöge, und Tugendreichen Fräuleins. Fr. Christina-Elisabetha von Bellingkhaussen. Welche Zoge, und Tugendreichen Fräuleins, Fr. Christina-Elisabetha von Bellingkhaussen, Welche
als erstlichen das S. Fräulein zu Riga den 17. Aug. im 7. Jahr ihres Alters, die S. Frau aber zu als erstlichen das S. Fräulein zu Riga den 17. Aug. im 7. Jahr ihres Alters, die S. Frau aber zu

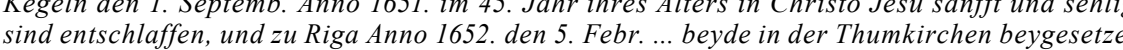
worden, auffgesetzt von guten Freunden (Dorpat: Johann Vogel, 1652), TLÜAR, XII-2620a.

21 Justa honoratissimae, piissimae omniq[ue] virtutum decore exornatissimae matronae Dorotheae von Wickeden, ... Dn. M. Erici Holstenii, Graec. \& Hebr. ling. in Academinal!] Gustaviana professoris ... conjugis, quae 1630. d. 20. Martij ... Dorpati nata. An. v. 1654. die in templo Dorpatensi D. Johanni 16. Jul ... commendata est. Ab amicis obitum hunc solute (Dorpat: Johann Vogel, 1654), TLÜAR, BA08/530 (Kopie). Das Original wird in der Königlichen Bibliothek Stockholm aufbewahrt.

22 Enn Küng, ,Johann Köhler ja Narva trükikoda 1695-1705“, Läänemere provintside arenguperspektivivid Rootsi suurriigis 16/17. sajandil, II (Tartu: Eesti Ajalooarhiiv, 2006), 
Narvaer Druckerei entstandene Druck - die Leichenpredigt des Jacob Lang bei der Beerdigung der Ehefrau des Kammerherrn von Narva, Ingermanland und Kexholm Erik Andersson Borg Catharina Törneroos, die 1696 in der Domkirche bestattet wurde. ${ }^{23}$

Ähnliche Totenschädelvignetten finden sich in den Werken sowohl schwedischer als auch finnischer Druckereien, z. B. in den Druckwerken der Stockholmer Drucker Peter von Selow (1633), Michael Laurelius $(1695,1697)$, Henrik Keyser $(1698,1701)$, Nils Wankijf und Johann Heinrich Werner (1688, 1692, 1694), Olaus Enaeus (1698, 1700), Georg Gottlieb Burchard (1694) und des in Uppsala wirkenden Druckers Henrik Curio (1674). Die Verwendung beginnt um 1630 und ist bis $1691 \mathrm{zu}$ beobachten. In Finnland sind ähnliche Vignetten in den Werken der Druckerei der Åbo Akademie und den Werken von Johannes Gezelius / Henrik Cristopher und Johan Christopher Merckell in Jahren 1675-1752 registriert worden. ${ }^{24}$

Mehrere verschiedene Vignetten mit dem Totenschädelmotiv schmücken die Trauerreden und -gedichte des letzten Tallinner Druckers der Schwedenzeit Christoph Brendeken (1649-1710, Wirkungszeit in Tallinn 1676-1710). Die Bibelsprüche „Sei getrost bis an den Tod, so will ich dir die Krone des Lebens geben“ (Offenbarung 2,10) oder "Selig ist der Mann, der die Anfechtung erduldet; denn nachdem er bewährt ist, wird er die Krone des Lebens empfangen, welche Gott verheißen hat denen, die ihn liebhaben" (Jk. 1,12) mit dem die Vignette bezeichnenden Motto "Assuesce et persevera“ (siehe Abb. 8) kann man sowohl auf dem Titelblatt, am Anfang als auch am Ende des Textes finden. In der estnischen Literaturgeschichte ist diese Vignette bekannt durch die Gedichtsammlung, die anlässlich des Todes der Ehefrau des Keilaer Pastors Anton Heiderich - Hedwig Sidonia Wagner (1628-1686) - erschienen ist. Diese Vignette wurde in den Jahren 1677-1692 benutzt, es ist nicht gelungen, ähnliche Vignetten

23 Jacob Lang, Gudz Barnas Wandring och Högsta Goda, Förestält då then Edle och MycketDygdesamma Matrona, Catharina Törneroos, Kongl. May.tz Tro Tienares och Borgz. Kärälskelige Maka, I Narvens Dom-Kyrckia den 29 Martii, Ahr 1696. uthi en förnähm och Folckrijk Försambling blef begrafwen (Narva: Johann Köhler, 1696), TLÜAR, BA08/531 (Kopie). Das Original befindet sich in der Königlichen Bibliothek Stockholm.

24 Drees, Deutschsprachige Gelegenheitsdichtung, 339, I.2; 343, III.1; 363, IV.81373, V.39; 386, V.50.U.; 388, VI.38; 405, VII.43; 416, IX.9; 438, XIV.6; Perälä, Suomen typografinen atlas, I, 214-215, TA.III.13-13a; 231, RTA.III.44; 233, TA III.50; Suomen typografinen atlas
II, 130-131, GM III. 5-5b.
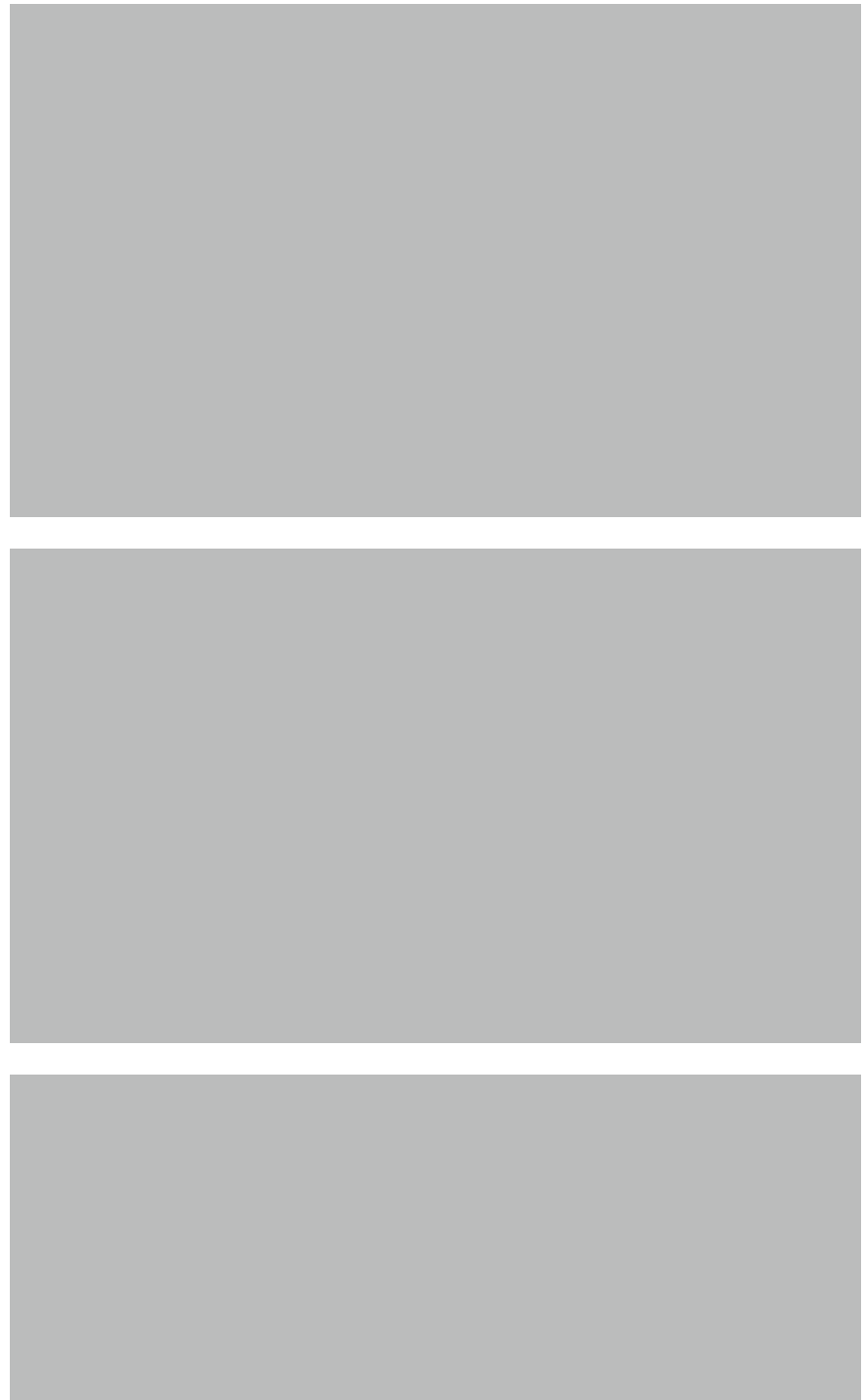

Abb. 8. TALLINN: Ausmass des Originals $55 \times 95 \mathrm{~mm}$. Den Schmertzlichen Hintritt Der Weiland Edlen, GroszEhr und Tugendbelobten Frauen, Fr. Hedwig Sidoniae Wagnerin ... (Reval: Christoph Brendeken, 1686).

Abb. 9. TALLINN: Ausmass des Originals $35 \times 60 \mathrm{~mm}$. Georg Günther Tunzelmann, Die Seligkeit der Gerechten nach dem Tode, bey dem Hochansehnlichen Leichen-Begängnis Des (.. Hn. Christian Buchau, der Stadt Reval Hochverdienten Altesten Herren Burgermeisters ...

Abb. 10. TALLINN: Ausmass des Originals $40 \times 110 \mathrm{~mm}$. Denkmal der Liebe, Hochachtung und Dankbarkeit welches bey dem unvermutheten Tode des ... Herrn Bürgermeisters, ... Präsidis und sämtlichen Stadt-Schulen Directoris Adrian Heinrich Frese... (Reval: Axel Heinrich Lindfors, 1779), TLÜAR, XII-306. 
in den schwedischen und finnischen Druckereien $\mathrm{zu}$ finden. In diesem Trauerdruck haben sechs Autoren ihr Beileid ausgedrückt. ${ }^{25}$

Die die Wange in die Hand stützende Figur mit der Darstellung von Totenschädel und Todesengel (siehe Abb. 9) hat in den Trauerschriften zu Beginn des 18. Jahrhunderts der Tallinner Drucker Christoph Brendeken in der dem Rektor des Tallinner Gymnasiums Herrn Michael Sigismundi (1645-1709) gewidmeten Trauergedichtsammlung ${ }^{26}$ verwendet und später auch der Drucker Johann Köhler; diese Vignette findet sich in den Drucken bis 1724.

In der ersten Hälfte des 18. Jahrhunderts treten Motive mit der Darstellung eines Totenschädels zugunsten von Sargmotiven in den Hintergrund. Die Darstellung eines Totenschädels tritt in den Trauerschriften von neuem in der zweiten Hälfte des 18. Jahrhunderts auf, als Axel Heinrich Lindfors (Wirkungstätigkeit ca. 1775-1784, vorher, d. h. 1769-1775, leitete die Druckerei anstelle des minderjährigen Sohnes der Vater, der Kaufmann Axel Heinrich Lindfors Sen.) als Buchdrucker in Tallinn tätig war, dann schon im Rokokostil, wobei der Totenschädel in Rosenzweige verflochten war. Totenschädelmotive der Lindfors'schen Druckerei sind in den Trauerschriften seit $1775 \mathrm{zu}$ beobachten.

Die in Rosenzweige eingewickeltes Gebein mit Totenschädel darstellende Vignette (siehe Abb. 10) schmückt als Kopfvignette den gedruckten Text der Kantate, die bei dem Begräbnis von Peter August Friedrich (1696-1775), Herzog von Schleswig Holstein Beck und Generalgouverneur von Estland, vorgetragen geworden ist. Als Schlussvignette wird in demselben Druck ein Totenschädel mit einer Rosenblüte, der sich auf einem an eine Perlmuschel erinnernden Untergrund befindet, dargestellt (siehe Abb. 11). ${ }^{27}$

25 Den Schmertzlichen Hintritt Der Weiland Edlen, GroszEhr und Tugendbelobten Frauen, Fr Hedwig Sidoniae Wagnerin, Des ... Herrn Anthonii Heiderichs, Treufleissigen Pastoris zu Kegel. ... Hertzgeliebten Eheliebsten, Welche den 22. Septembr. 1686. zu Kegel Christlich beerdige ward; Betraurten Nachfolgende (Reval: Christoph Brendeken, 1686), TLÜAR, I-3393b (23). 26 Lacrumae gymnasii in obitu luctuosissimo praecellentissimi atq[ue] amplissimi domini, Dn. nec non SS. theol. \& linguae Hebraeae profess. meritissimi, d. XIX. Maji anno MDCCIX in templo D. Olai solenni more tumulati fusae (Reval: Christoph Brendeken, 1709), TLÜAR, XII-2937.

27 Cantate, am Begräbnistage Sr. Herzogl. Durchlaucht Herrn, Herrn Peter August Friedrich des Heil. Römischen Reichs, Fürsten, Erben zu Norwegen, der Stormarn und Ditmarsen, Herzogen zu Schlesswig Hollstein Beck, Grafen zu Oldenburg, und Delmenhorst, Ihro Russisch-Kayser. Majest. Hochgebietenden General-Feld-Marschalls, General-Gouverneurs des Herzogthum Alexander Newsky und St. Annen Ordens, Ritters, etc, etc. etc musikalisch aufgefïhret in Reval am 18 Merz 1775 (Reval: Axel Heinrich Lindfors, 1775), TLÜAR, XII-2998.
Die Vignette mit Darstellung eines Totenschädels mit einer Rosenblüte wurde als Dekorativelement bis zum Ende des 18 Jahrhunderts als Titel-, Kopf- und Schlussvignette verwendet.

\section{DER TOD ALS SENSENMANN}

In den selben Zeitraum, in dem auf der Vignette der Tod als Krone des Lebens dargestellt ist, fällt auch eine andere, in der Druckerei von Christoph Brendeken gedruckte Trauerdrucke verzierende Vignette mit der Devise der Zwangsläufigkeit des Sterbens „Nemo hic excipitur“ (siehe Abb. 12). Die in den Jahren 1689-1707 als Kopf vignette die Trauerdrucke verzierende Vignette stammt wahrscheinlich aus dem deutschen Sprachraum; dieselbe Vignette ist auch in einer in Wismar erschienenen Leichenpredigt beschrieben worden. ${ }^{28}$ In den Werken finnischer und schwedischer Druckereien ist die Vignette nicht beschrieben worden.

Bei den Symbolen des Todes kommt zu Totenschädel und Stundenglas das Motiv des Todesengels hinzu - der Sensenmann symbolisiert das Durchtrennen des Lebensfadens. Dieselbe visuelle Bedeutung trägt die Vignette, die die Leichenpredigt Bischof Joachim Salemanns (1629-1701, Tallinner Superintendent 1673-1691, Bischof von Estland 1693-1701) verziert (siehe Abb. 13). ${ }^{29}$ Die gleiche Vignette war auch in der Tartuer Buchdruckerei in Gebrauch. ${ }^{30}$

28 Albert Krüger, Insignia Christianorum Welt-müder und Himmels-begieriger Christen Geistliches Wapen: Welches bey Christ-Adlicher und Hoch-trauriger Leichbegängniß Des ... 9 und 10 . Uhren/fein sanfft und gantz seelig im Herrn entschlaffen/ und den 31 . Augusti in der Kirchen zu Beydendorff mit Christ-Adlichen aund ansehnlichen Leich-Ceremonien beygesetzet worden/Aus den Worten des 73. Psalms: Herr/wenn ich nur dich habe/ so frage ich nichts nach worden/ Aus den Worten des 73. Psalms: Herr/ wenn ich nur dich habe/ so frage ich nichts nach
Himmel und Erden/ u.s.w. vorgestellt Auch auff inständigs und vielfätiges Begehren zum Abdruck außgefertiget Albertus Krüger/ Past. zu Beydendorff (Wissmar: Jochim-Georg Rheten, 1670). Die Vignette befindet sich auf S. 80, unten im Chronogramm ist das Erscheinungsdatum 1670 . ALLes ThVn LäVffet nVn zVM EnD. Volltext: https://gdz.sub.uni-goettingen.de/id/PPN62295 0509?tify $=\{\% 22$ view\%22:\%22info\%22\} [zuletzt aufgerufen am 15.07.2018].

29 Justus Blankenhagen, Idea boni episcopi, oder Der unsträffliche Bischoff. Nach dem Beispiel des erleuchten Heiden Lehrers Pauli, auss der 11. Epist. an die Corinth. am 1. Cap. Reval hochverdienten Bischoff. .... Nachdem Derselbe am 3. Martii dieses Jahres 1701. sanfft und seelig im Herren entschlaffen, und dessen verblichener Cörper den 20 sten Tag selbigen Monats, ... in der Königl. Dohm-Kirche, zur Erden bestattet. In einer Christlichen Leichpredig vorgestellet von M. Justo Blanckenhagen. Past. Reval. Primar (Reval: Christoph Brendeken, 1701), TLÜAR, I-3393b(7).

30 Jaanson, Tartu Ülikooli trükikoda 1632-1710, 144. 

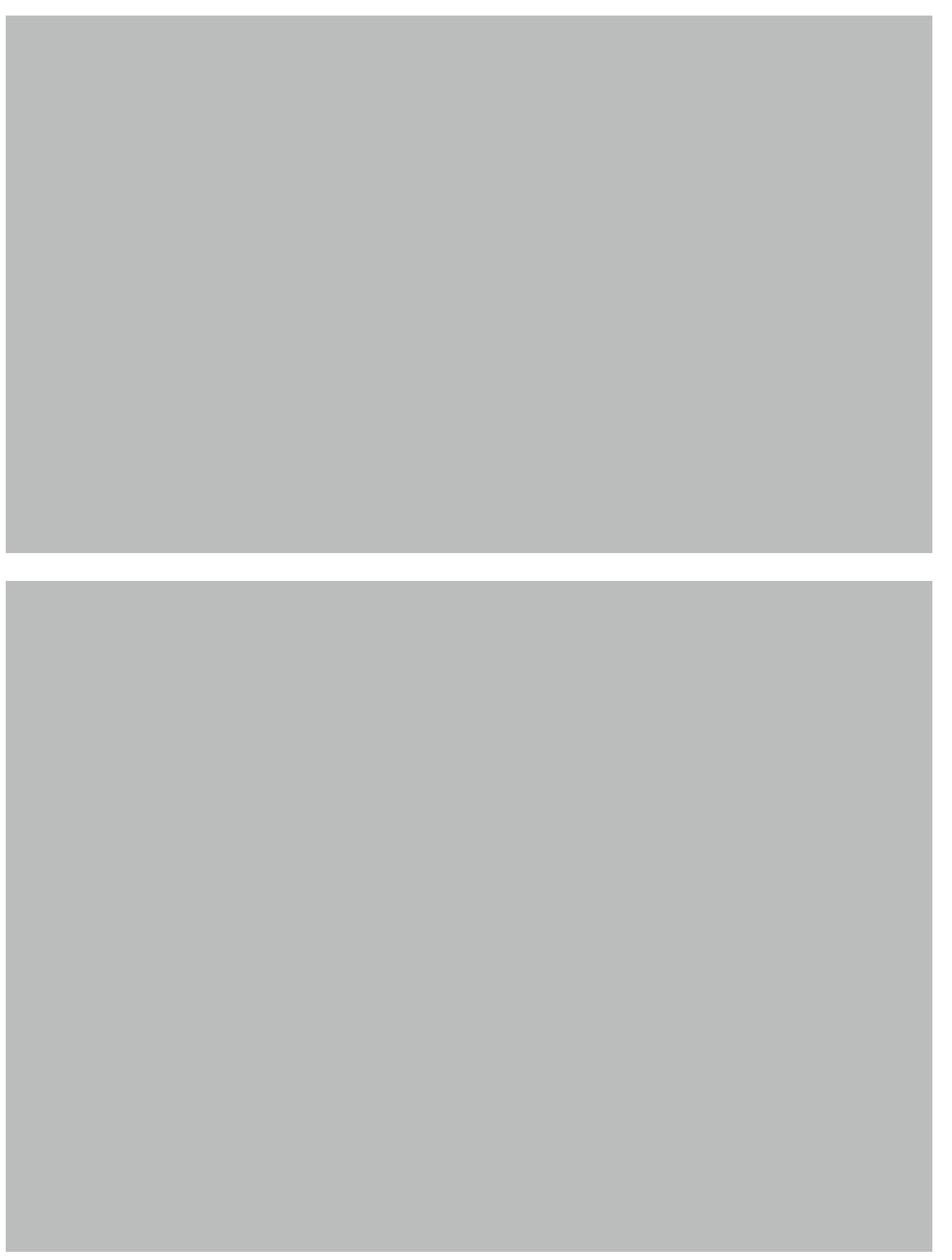

Abb. 11. TALLINN: Ausmass des Originals $35 \times 65 \mathrm{~mm}$. Ernst August Wilhelm Hörschelmann, Standrede bey der Beerdigung des Hochedelgebohrnen und Hochgelahrten (Reval: Axel Heinrich Lindfors, 1779).

Abb. 12. TALLINN: Ausmass des Originals $80 \times 115 \mathrm{~mm}$. Honoris ac amoris monumentum viro, dum viveret, plurimum reverendo atq[ue] praeclarissimo Dn. M. Christiano Hoppio... (Reval: Christoph Brendeken, 1689), TLÜAR, I-3393b(3).
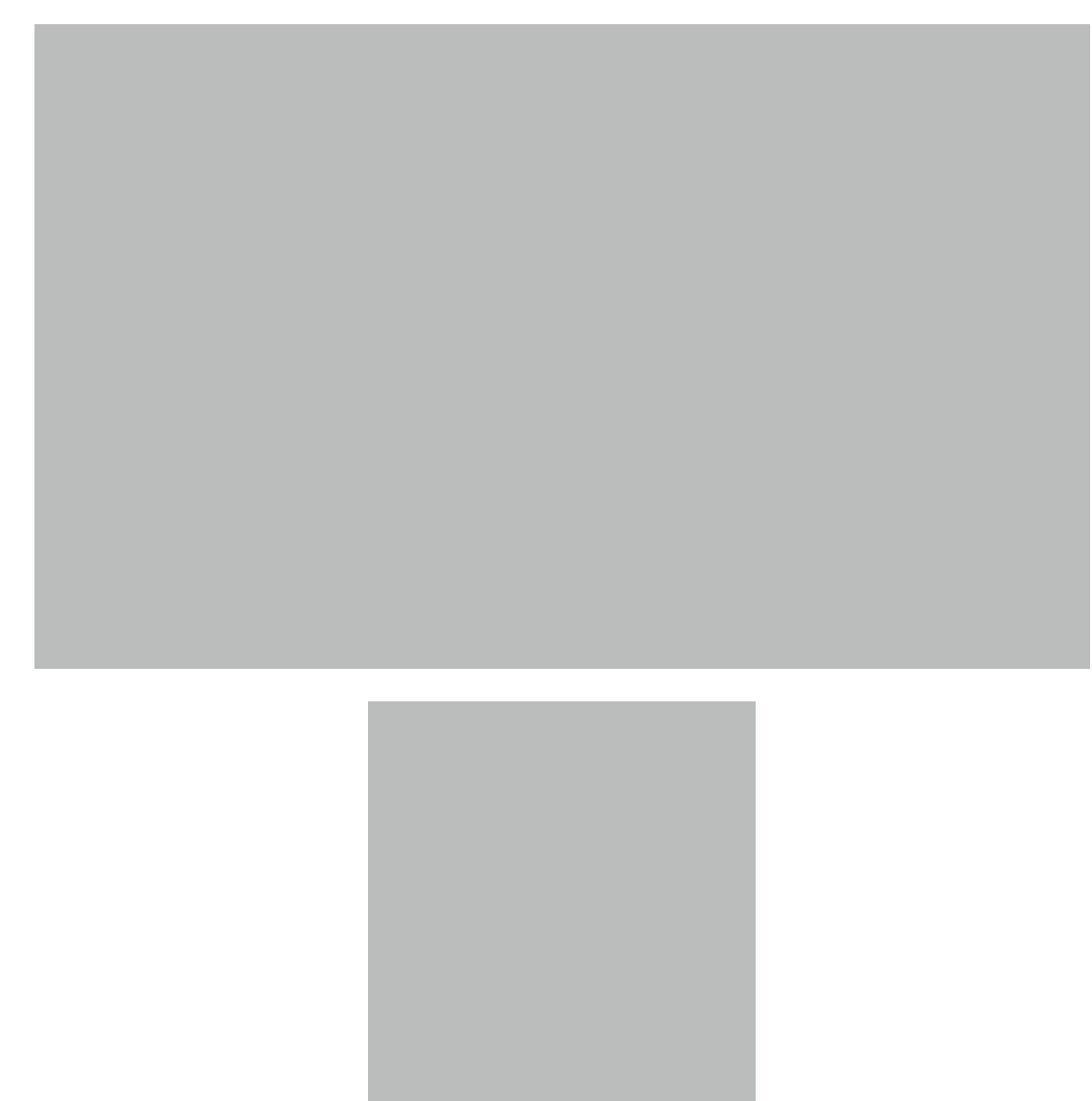

Abb. 13. TALLINN: Ausmass des Originals $55 \times 95 \mathrm{~mm}$. Justus Blanckenhagen, Idea boni episcopi, oder Der unsträfliche Bischoff. Nach dem Beispiel des erleuchten Heiden Lehrer Pauli... (Reval: Christoph Brendeken, 1701).

Abb. 14. TALLINN: Ausmass des Originals $30 \times 35 \mathrm{~mm}$. Helden Arm und Stücken Blitzen Kan auch vor dem Tod nicht schützen... (Reval: Christoph Brendeken, 1678).

Die Personifikation des Todes als Skelett, mit der Sense in der einen und dem Stundenglas in der anderen Hand (siehe Abb. 15-16) - ist nach meinem Wissen als Vignette nur in einem Druck, in der bereits genannten Trauergedichtsammlung anlässlich des Todes der Ehefrau Hedwig Sidonia Wagner, benutzt worden.

Sowohl in Finnland als auch in Schweden ist diese Vignette in zahlreichen Trauerdrucken verwendet worden. Ein Vergleich zeigt, dass der in Tallinn benutzte Druckstock im Spiegelbild eine 

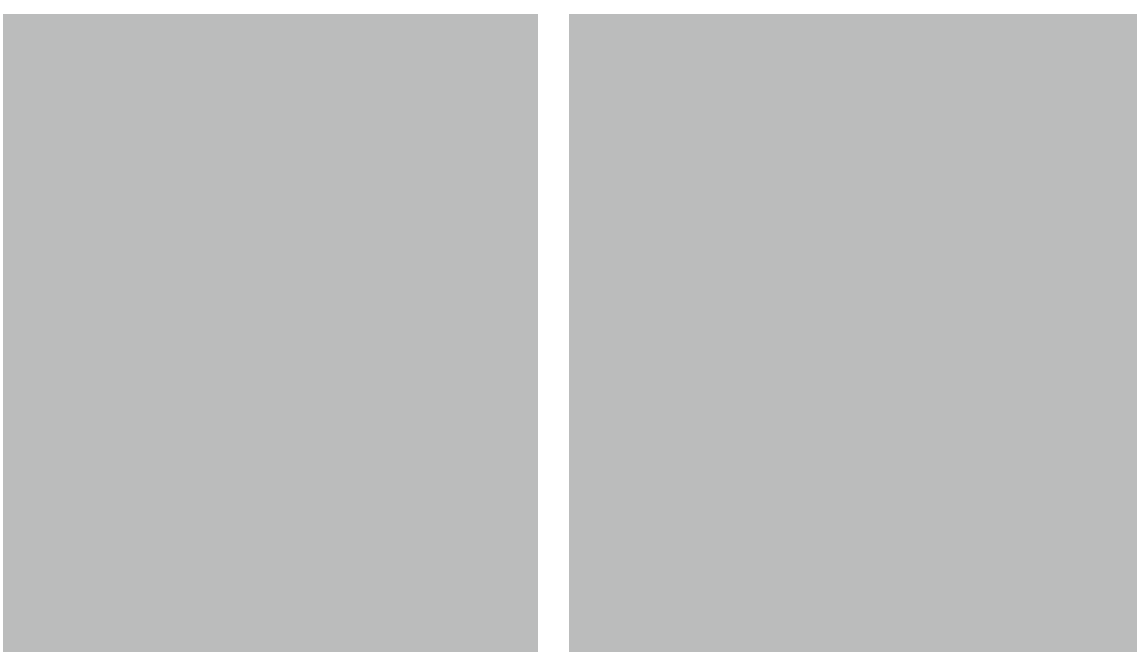

Abb. 15. TALLINN: Ausmass des Originals $75 \times 60 \mathrm{~mm}$. Den Schmertzlichen Hintritt Der Weiland Edlen, GroszEhr und Tugendbelobten Frauen, Fr. Hedwig Sidoniae Wagnerin... (Reval: Christoph Brendeken, 1686).

Abb. 16. Anna Perälä, Suomen typografinen atlas = Finsk typografisk atlas = Typographischer Atlas Finnlands, II (Helsinki: Helsingin Yliopiston Kirjasto, 2000), 129, GM III.2.

Darstellung des in den Nachbarländern verwendeten ist. ${ }^{31}$ Die in den schwedischen Druckereien verwendete Vignette, auf der der Tod mit der Sense in der Hand auf dem Sarg sitzt, hat man in den Tallinner Drucken nicht gefunden. ${ }^{32}$

Das Motiv des Todes als Sensenmann kommt in der ersten Hälfte des 18. Jahrhunderts auch in Kopfleisten samt der Mahnung „Hodie mihi cras tibi" vor (siehe Abb. 17). Erstmalig wurde die Vignette im bereits erwähnten Trauerdruck an Sigismundi, gedruckt in der Druckerei von Christoph Brendeken, verwendet. In den Druckwerken von Johann Köhler (1662-1736, Wirkungszeit in Tallinn 1716-1736) und seinem Sohn Jacob Johann Köhler (1698-1757, Wirkungszeit 1736-1757) ist die Vignette von 1720 bis 1739 registriert. Die gleiche Vignette, jedoch mit dem Todesengel im Spiegelbild, etwas anderer Hintergrundgestaltung und der Überschrift "Nemo hic excipitur"

31 Drees, Deutschsprachige Gelegenheitsdichtung, 350, IV.26; 368, V,12; 380, VI.1, VI.2; 438, XIV.5; Perälä, Suomen typografinen atlas, I, 208-209, TA.III.5; Suomen typografinen atlas, II, 128-129, GM.III.1-2.

32 Ibidem, 356, IV.51; 399, VII.17.
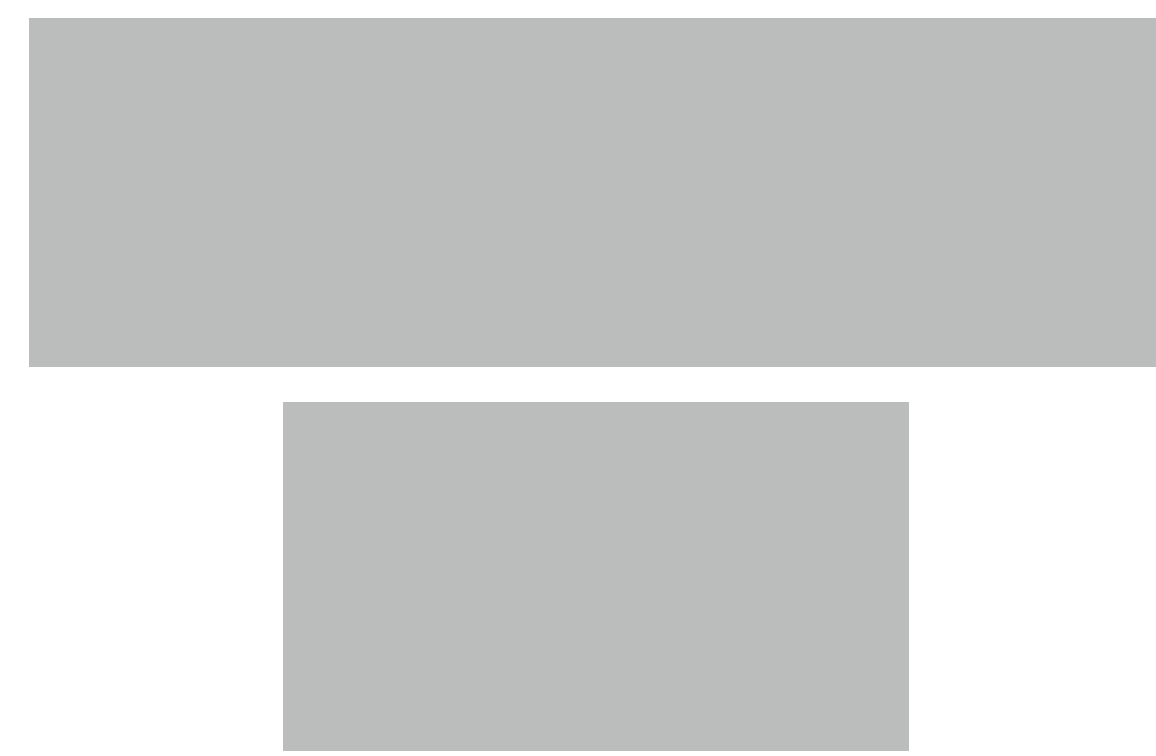

Abb. 17. TALLINN: Ausmass des Originals $40 \times 150 \mathrm{~mm}$. Georg Günther Tunzelmann Bei Tugendt und mit Ehren alten Muss Leben, auch im Tod', erhalten! Welches bei dem sehligen Hintritt Der Edlen GrossEhr und Tugendsahmen Frauen Fr. Gerdrutha Paulsen ... Abb. 18. TALLINN: Ausmass des Originals: $25 \times 55$ mm. Axel Friedrich Lindfors, Dem Andenken des Herrn Christian Niklas Jaenisch gewidmet ... (Reval: Axel Heinrich Lindfors, 1776), TLÜAR, XII-2990.

hat Anna Perälä in den Drucken des ausgehenden 17. Jahrhunderts und der ersten Hälfte des 18. Jahrhunderts der Druckerei der Aboer Akademie beschrieben. ${ }^{33}$

Das in Europa weit verbreitete Trauermotiv, in dem Flügel, Stundenglas und Sense das Scheiden aus dem Leben symbolisieren (siehe Abb. 18), ${ }^{34}$ kommt in den Tallinner Drucken erstmalig im Jahre 1776 vor und ist bis zum Jahr $1828 \mathrm{zu}$ verfolgen. Anfang des 19. Jahrhunderts schmückt diese Vignette vorwiegend die für Begräbnisfeiern gedruckten Gesangblätter.

33 Perälä, Suomen typografinen atlas, I, 308-309, TA.XI.1-1b.

34 Dieselbe Vignette findet man als ein Beispiel in dem Praktischen Handbuch der Buchdruckerkunst für Anfänger (Leipzig, 1791) von Christian Gottlob Täubel. 


\section{DIE DARSTELLUNG DES SARGES IN FUNERALDRUCKEN}

Neben dem Totenschädel ist der Sarg ein häufiges, die Trauerdrucke verzierendes Motiv. Die Darstellung eines Sarges ist in ganz Europa üblich gewesen und auch in Estland gedruckte Schriften kommt der Sarg in mehreren Varianten vor. Das Sargmotiv war in den gedruckten Leichenpredigten und Gedichtsammlungen ein ganzes Jahrhundert vom Beginn der Drucktätigkeit in Estland bis zur Mitte des 18. Jahrhunderts in Gebrauch.

Die erste Vignette mit der Darstellung eines Sarges ist derselbe Druckstock, den Christoph Reusner schon in seiner Stockholmer Zeit für seine Werke verwendet hat: ein schwarzes Leichentuch mit einem weißen Kreuz auf dem Sarge (siehe Abb. 19). Als Titelvignette haben diese Darstellung ausnahmslos alle Tallinner Buchdrucker von 1635 bis 1708 benutzt. Sogar das Gedicht, das zum Andenken an den Buchdrucker Christoph Reusner gedruckt wurde, verziert die Vignette mit dem Sarg. ${ }^{35}$ Eine ähnliche Vignette findet sich oft auch in schwedischen und finnischen Druckschriften. ${ }^{36}$

Zeitlich folgend (registriert in im Zeitraum 1642-1661 erschienenen Drucken) ist die Vignette mit Sarg und Wappenschild, auf der der Name und das Todesjahr des Verstorbenen verzeichnet ist. Diese Vignette tritt nur in den Begräbnisschriften weniger Verstorbener von hohem Rang auf, so z. B. der Pastor der St. Nikolaikirche Eberhard von Rentelen, Maria von Delwig sowie die Ehefrau des Generalgouverneurs Von Ingermanland und Kexholm Christer Horn, Görel Sparre (siehe Abb. 20)

Die Vignette mit Wappenschild kann man auch in den Personaldrucken der Stockholmer Buchdrucker Ignatius Meurer und Johann Georg Eberdt (1640, 1641, 1660-1678), Henrik Keyser (16341636) und Nils Wankijf (1680) finden. Als einziges Beispiel aus dem finnischen Territorium ist eine Vignette in einem Wiborger Druck aus dem Jahre 1695 bekannt. ${ }^{37}$

35 Lob vnd Vrsprung der Löblichen Buchdrucker-Kunst: Zu Ehren geschrieben bey dem Begräbnüs Dess Weiland Erbarn, Kun[stre]ichen vnd wolverdienten Buchdruckers H. Christoff Reusners, desz ältern; Welcher Anno 1637. den 20. Augusti zu Revall in Gott seelig entschlaffen, vnd den 24. dieses hernach Christlich zu Grabe begleitet ist (Reval. Reusners Witwe, 1637). Da einzige uns bekannte Exemplar befindet sich in der Ratsschulbibliothek Zwickau.

36 Drees, Deutschsprachige Gelegenheitsdichtung, 339, 1.3; 349, IV.18; 370, V.25.

37 Ibidem, 347, IV.7., IV.9, IV.48;. 366, V.2; Perälä, Suomen typografinen atlas, II, 416-417, WIB.II.7
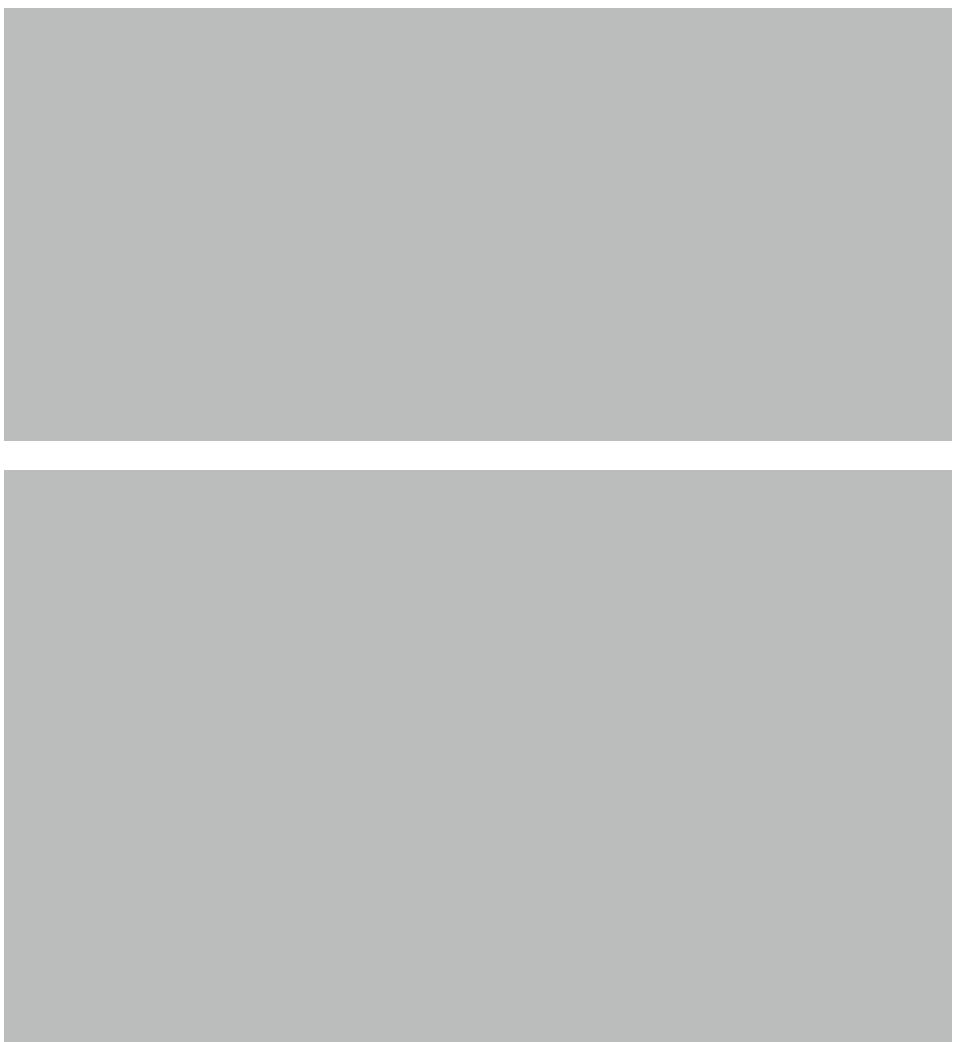

Abb. 19. TALLINN: Ausmass des Originals $35 \times 75$ mm. Isaac Hasselblatt, Hertzliche Trauer-Klage über den allzufrühzeitigen Hintritt Der ... Frauen Dorothea Elisabeth Hertzogin ... (Reval: Christoph Brendeken, 1696), TLÜAR, I-5443(27).

Abb. 20. TALLINN: Ausmass des Originals $70 \times 110 \mathrm{~mm}$. Justus Heinrich Oldekop Frommer Christen Frewd im Leid, ... Als ... Fr. Görel Sparre, ... zu ihrem Erb-Begräbniss, nach der Koniglichen Residenz-Stadt-Stockholm abgeführet werden solte (Reval: Adolph Simon, 1661), TLÜAR, I-3389b(12).

In der Mitte des 17. Jahrhunderts begannen Darstellungen von Totenschädel, Sanduhr und Kruzifix mit oder ohne Bibelspruch den Sarg zu verzieren. Die Vignette mit Sarg, Stundenglas und Engelsflügeln (siehe Abb. 21) ist in Tallinner Drucken aus den Jahren 1659-1683 und in Tartuer Drucken aus den Jahren 1696-1710 registriert worden. ${ }^{38}$

38 Jaanson, Tartu Ülikooli trükikoda 1632-1710, 143. 


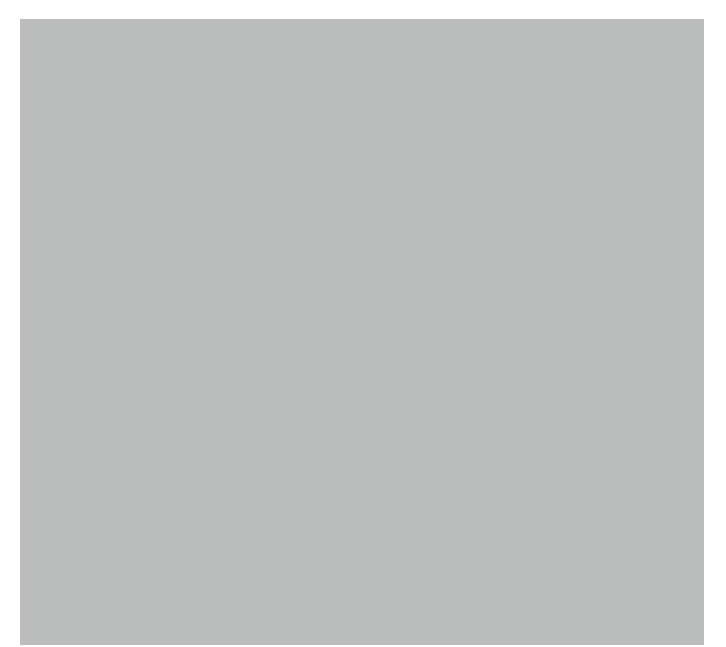

Abb. 21. TALLINN: Ausmass des Originals $51 \times 60 \mathrm{~mm}$. Joachim Sellius, Vox coelica eine Stime vom Himmel, ... Bey ansehnlicher und Volckreicher Leich-Begängnüss Des ... Herrn M. Isaaci Mariaestadii ... (Reval: Christoph Brendeken, 1682), TLÜAR, I-3393b(12).

Die Darstellung mit Sarg, Schädel und der Sentenz „Hodie mihi cras tibi“ finden wir in Tallinner Drucken aus den Jahren 1658-1696. ${ }^{39}$ Die hauptsächlich im Kopf des Textes vorkommende Vignette ist manchmal auf einer separaten Seite mit aus verschiedenen Ziertypen dargestellte Leisten gedruckt (siehe Abb. 22). ${ }^{40}$

Aus den Drucken der Pärnuer Periode der Academia Gustavo-Carolina sind aus den ersten Jahren des 18. Jahrhunderts auch Darstellungen des Sarges unter einem Baldachin in den Trauerdrucken bekannt (siehe Abb. 23). ${ }^{41}$

39 Vgl. Perälä, Suomen typografinen atlas, I, 206-207,TA.III.3-3a.

40 Gabriel Elvering, Condolentz Sermon Uber der geschwinden Volkommenheit Des Weiland Ehrenvesten Gesellen Arend Johan Badens, Welcher den 1. Augusti Anno 1657. zu Kirrisal in Jerven seelig entschlaffen, und darauff in folgendem Jahr den 14. Februarij zur Erden bestätiget in seinem Erb-Begräbnüss zu Reval, Gehalten in Kesal den 30. Augusti, Anno 1657. und auff.

Begehren zum Druck gefordert Von G. E.P.P. (Reval: Adolph Simon, 1658), TLUAR, I-5443.
$41 \quad$ Trauer-Arien Welche Bey der Beerdigung des HochEhrwürdigen, Gross-Achtbahren und Hochgelahrten Herrn, Herrn Martini Westphals, Weyland treufleissigen Ober-Pastoris be der hiesigen Stadt Pernau und Inspectoris der Schulen, Als Derselbe Nach seinen seel. Hintritt aus diesen[!] Leben zu seiner Ruhe-Kammer gebracht ward. Aus schuldiger Pflicht gegen den Hochseel. Verstorbenen als liebgewesenen Freund und hohen Gonner, wie auch gegen seine hinterlassenen hochbetrübten wehrten Angehörigen, Kürzlich und einfältig haben entwerffen sollen Untenbenandte (Pernau. Johan Brendeken, 1702), TLÜAR, BAC 13/324 (Kopie). Da einzige uns bekannte Exemplar befindet sich in der Akademischen Bibliothek der Universitä
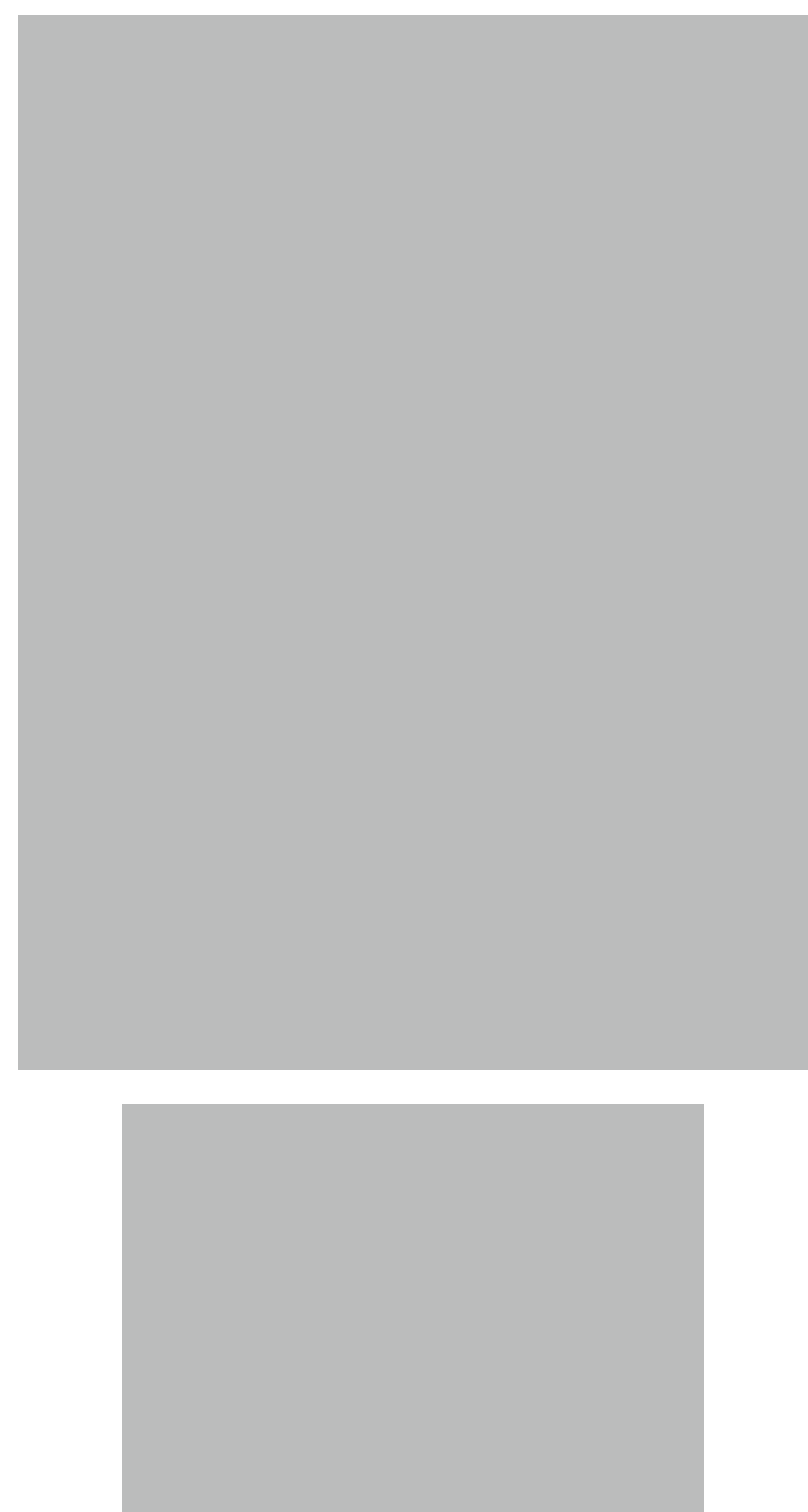

Abb. 22. TALLINN: Ausmass der Vignette $45 \times 65 \mathrm{~mm}$. Gabriel Elvering, Condolentz Sermon Uber der geschwinden Volkommenheit Des Weiland Ehrenvesten Gesellen Arend Johan Badens... (Reval: Adolph Simon, 1658).

Abb. 23. PÄRNU: Ausmass des Originals $75 \times 120 \mathrm{~mm}$. Trauer-Arien Welche Bey der Beerdigung des HochEhrwürdigen, Gross-Achtbahren und Hochgelahrten Herrn, Herrn Martin Westphals... (Pernau: Johann Brendeken, 1702). 

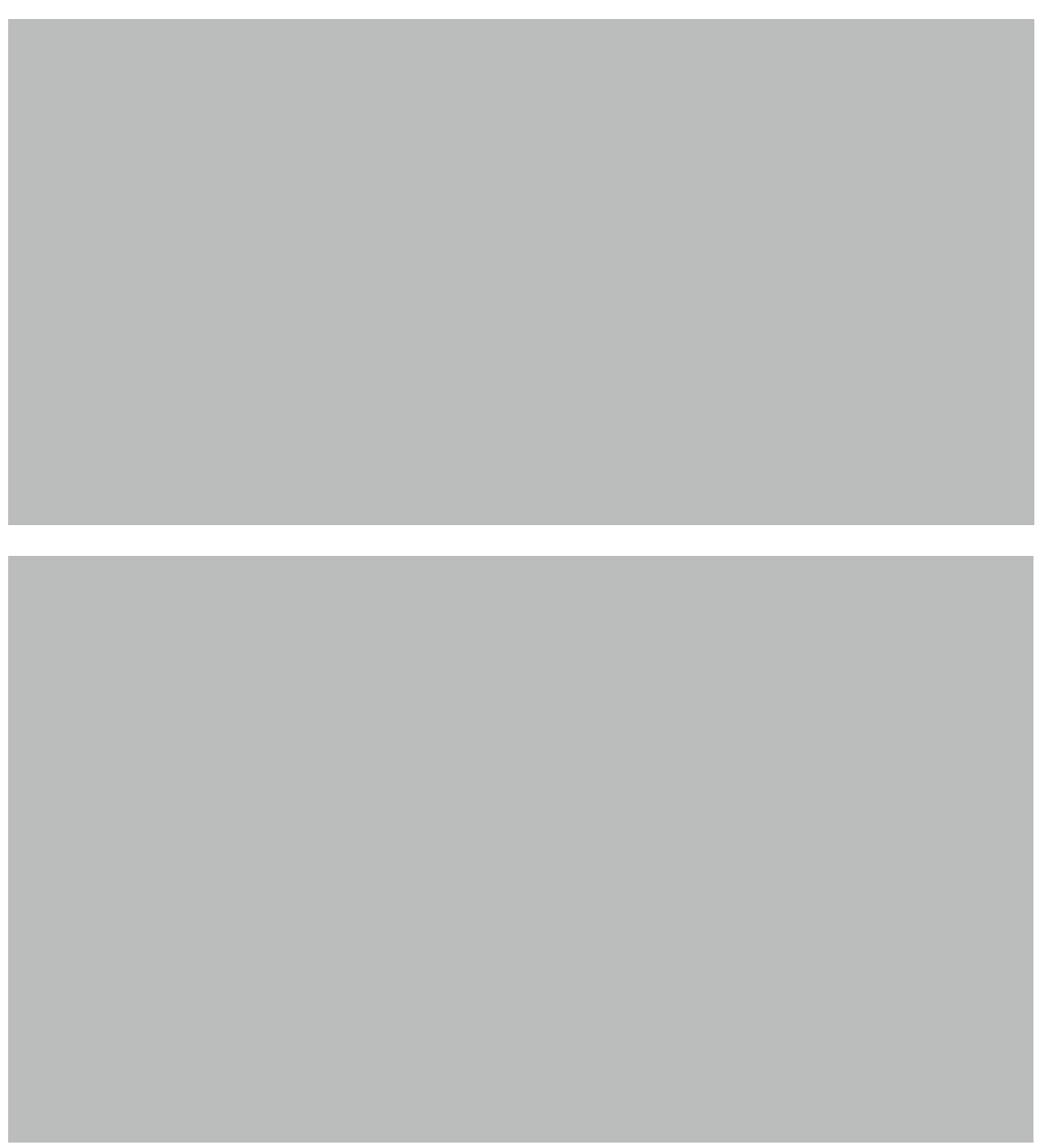

Abb. 24. TALLINN: Ausmass des Originals $65 \times 145 \mathrm{~mm}$. Den rätta Süllheeten fördold här under Korset $i$ Hoppet... (Reval: Christoph Brendeken, 1682).

Abb. 25. TALLINN: Ausmass des Originals $55 \times 105 \mathrm{~mm}$. Trauer- und Trost-Worte, über den zwar frühzeitigen doch seeligen Abscheid auss dieser Welt... (Reval: Christoph Brendeken, 1706).

Damalige schwedische und finnische Gelegenheitsschriften enthalten den Sarg unter einem Baldachin in mehreren verschiedenen Varianten, ${ }^{42}$ exakt dieselbe Vignette hat man aber nicht registriert.

In den während des Nordischen Krieges erschienenen Drucken kann man eine Vignette finden, wo ein auf den gekreuzten

42 Drees, Deutschsprachige Gelegenheitsdichtung, 352, IV.33; 397, VII.12; 406, VII.48; 412 , VIII.8; 439, XIV.8; Perälä, Suomen typografinen atlas, I, 216-217, TA.III.15-15a; Suomen typografinen atlas, II, 138-139, GM.III.14.
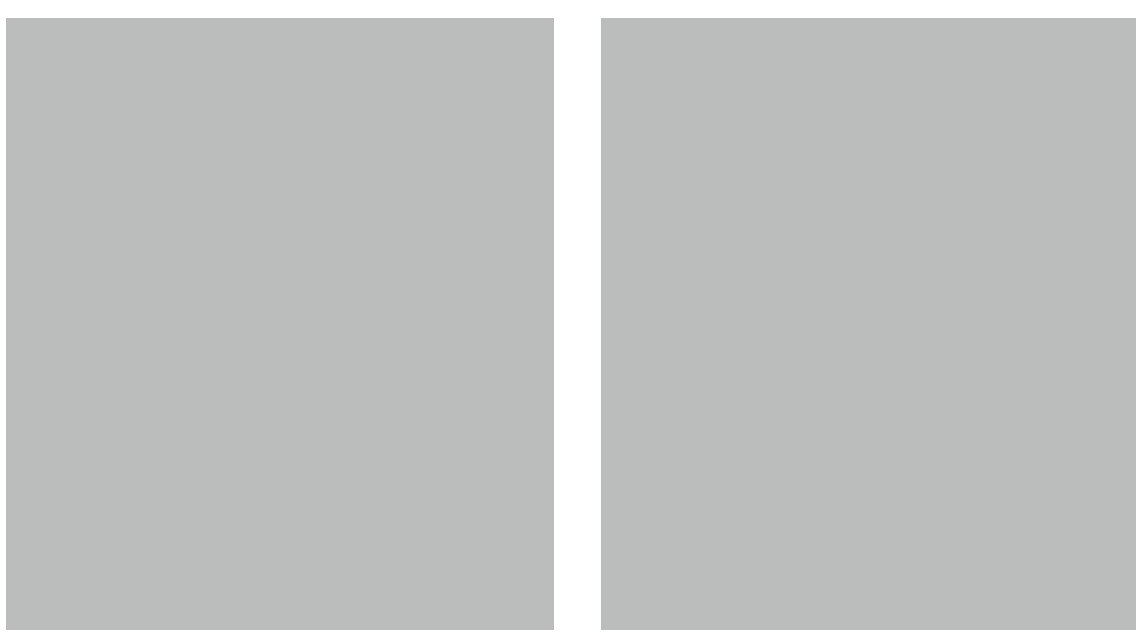

Abb. 26. TALLINN: Ausmass des Originals $35 \times 30 \mathrm{~mm}$. Bey der Beerdigung der Wohlseligen Frau Räthinn Maria von Dehn... (Reval: Johann Gottfried Minuth, 1801).

Abb. 27. TALLINN: Ausmass des Originals $35 \times 30 \mathrm{~mm}$. Axel Friedrich Lindfors, Dem Andenken des Herrn Christian Niklas Jaenisch... (Reval: Axel Heinrich Lindfors, 1776).

Oberschenkelknochen ruhender Schädel auf dem Boden zwischen Blumen liegt und der mit einem Leichentuch bedeckte Sarg mit der Aufschrift „Folg(e) mir nach“ (Mk 10, 21) sowie ein Kreuz im Hintergrund $\mathrm{zu}$ sehen sind (siehe Abb. 24). Ebenso eine Vignette kommt auch in finnischen und schwedischen Drucken vor, doch wie bei der Vignette mit dem Skelett, hat man es in den Tallinner Drucken mit einem Spiegelbild zu tun. ${ }^{43}$

In den Tallinner Leichenpredigten und Gedichtsammlungen der ersten Hälfte des 18. Jahrhunderts gibt es auch das Kruzifix als Verzierungselement des Sarges (siehe Abb. 25). Aus den Jahren 1706-1756 sind bisher drei Druckschriften mit solch einer Vignette verzeichnet.

Die Kruzifixvignetten kommen auch in finnischen Gelegenheitsdrucken vor (es fehlen als Vergleichsmaterial Angaben zu den sich in schwedischen Drucken der ersten Hälfte des 18. Jahrhunderts befindenden Verzierungen). ${ }^{44}$

43 Vgl. Drees, Deutschsprachige Gelegenheitsdichtung, 351, IV.29; Perälä, Suomen typografinen atlas, I, 208-209, TA.III.7; Suomen typografinen atlas, II, 132-133, GM.III.7-7a.

44 Perälä, Suomen typografinen atlas, I, 218-219, TA.III.20; 224-227, TA.III.35, 35 a, 39, 40; 232-233, TA.III.47; Suomen typografinen atlas, II, 138-141, GM.III.13, 13a, 14, 15, 15a 
In der zweiten Hälfte des 18. Jahrhunderts verschwinden die Sargdarstellungen auf den Vignetten. Zur vorherrschenden Vignette wird eine kleine Vignette mit Sanduhr, Sense und Flügeln. Als Titelvignette und als Verzierung der Initiale verwendete der Tallinner Buchdrucker Lindfors eine Vignette mit Kreuz- und Totenschädeldarstellung. Im Falle der Verzierung des Anfangsbuchstabens ist die Initiale in der Mitte eines Blumenkranzes platziert (siehe Abb. 26-27).

In den Drucken ist diese Vignette von 1776 bis $1809 \mathrm{zu}$ finden, zu Beginn des 19. Jahrhunderts verzierte sie gewöhnlich die für die kirchliche Begräbnisfeier gedruckten Gesangblätter.

\section{DIE VERBREITUNG DEKORATIVER DRUCKSTÖCKE}

Der Vergleich mit schwedischen und finnischen Quellen zeigt, dass ähnliche Vignetten und ihre verschiedenen Varianten fast zeitgleich in unterschiedlichen Gebieten und bei vielen Buchdruckern in Gebrauch waren. Dies wirft wiederum die Frage auf, wie die Vignetten hergestellt und verbreitet wurden. Es gibt dazu wenig Untersuchungen, ebenso sind nur wenige Produktionskataloge der Schriftgießereien aus dem 17. und 18. Jahrhundert erhalten..$^{45}$ Es ist anzunehmen, dass zu der Herstellung von Metalllettern recht bald die Herstellung von Ziertypen und dekorativen Druckstöcken und der Handel mit denselben hinzukam. Nach Jan Drees wurden in Europa schon im 16. Jahrhundert Zierinitialen und Ziertypen aus Metall hergestellt. ${ }^{46}$ Die Druckstöcke der größeren Bildinitialen, Zierleisten und Vignetten wurden anfänglich in der Holzschnitttechnik gefertigt, doch die Herstellung von Abgüssen der Druckstöcke ermöglichte die Vervielfältigung der Vignetten und Zierleisten sowie deren Verkauf. ${ }^{47}$ In seinen Erklärungen, wie die Abgüsse der Holzdruckstöcke angefertigt wurden, stützt sich Jan Drees auf die in der ersten Hälfte des 19. Jahrhunderts erschienenen Werke von Carl Friedrich

45 Drees erwähnt einen Schriftproben-Katalog des Leipziger Druckers Gregorius Ritzsch (1584-1643); der Katalog wurde im Zweiten Weltkrieg zerstört: Drees, Deutschsprachige Gelegenheitsdichtung, 264

46 Ibidem, 257

47 Ibidem, 257, 261-262 von Ruhmor und Bernhard Ringelhardt. ${ }^{48}$ Die Herstellungs- und Vervielfältigungstechnologie von Druckstöcken ist dadurch mit großer Wahrscheinlichkeit rekonstruierbar, doch ihre Verbreitungsund Vermarktungsweisen erfordern weitere Untersuchungen.

Die einzige Beschreibung einer Einrichtung einer Druckerei des letzten Viertels des 17. Jahrhunderts finden wir im Anhang der Geschichte der Buchdruckkunst in Riga von Arendt Buchholtz. Im Oktober 1683 starb der Rigaer Buchdrucker Heinrich Bessemesser und der neue Inhaber der Stadtdruckerei wurde Georg Mattias Nöller. In Verbindung mit dem Besitzerwechsel wurden die Bestände der Druckerei am 18. Juni 1864 aufgenommen. Im Verzeichnis steht über die für die Begräbnisdrucke benutzten Vignettendruckstöcke folgendes: 1 Großer Leichenstock, worauf stehet Nemo hic excipitur, 1 Cum signo: Christus ist mein Leben, 1 Spes alterae vitae, 1 Todten Kopf mit 3 Blumen, 1 Stock, worauff: Sic itur ad astra mit einem Stund-Glass manquiret. ${ }^{49}$

Hinsichtlich der Einrichtung estnischer Buchdruckereien im 17. und 18. Jahrhundert haben wir keine Urkunden gefunden. Bei der Analyse der in den Werken der Tartuer Buchdruckerei vorkommenden Vignetten stützt sich Ene-Lille Jaanson auf die Forschungen von Jan Drees, Horst Kunze und Hugo Lagerström. ${ }^{50}$ Der einzige erhaltene Katalog von Letter-, Zierleisten- und Vignettenbeispielen der Tallinner Stadt- und Gymnasialdruckerei stammt aus der Zeit Anfang des 19 Jahrhunderts. ${ }^{51}$

Die einzige Möglichkeit, eine Vorstellung von den in der Frühen Neuzeit in den damaligen gedruckten Werken verwendeten Dekorativelementen $\mathrm{zu}$ bekommen, ist die heute erhaltenen Druckschriften zu untersuchen. Dies nimmt viel Zeit in Anspruch und ist mühselig, denn oft befinden sich die einzigen erhaltenen

48 Drees, Deutschsprachige Gelegenheitsdichtung, 265-268: Carl Friedrich Rumohr, Zur Geschichte und Theorie der Formschneidekunst (Leipzig, 1837); Bernhard Ringelhardt, Die Kunst alle Arten Abgüsse und Abdrucke von Münzen, Medaillen, Cameen, Glasplasten, Kafern, Metall, Glas, Thon. Holzmassen etc. Auf's sauberste und vollkommenste zu verfertigen, nebst Anweisung zum Abklatschen und Beschreibung der neuesten französischen Clichirmaschinen (Quedlinburg, Leipzig: Basse, 1834).

49 Arend Buchholtz, Geschichte der Buchdruckerkunst in Riga, 1588-1888: Festschrift der Buchdrucker Rigas zur Erinnerung an die vor 300 Jahren erfolgte Einführung der Buchdruckerkunst in Riga (Riga: Müller, 1890), 358-360. Dasselbe Inventar der Rigasche Buchdruckerei haben Christa Pieske und Jan Drees aufgezeichnet.

50 Jaanson, Tartu Ülikooli trükikoda 1632-1710, 91-97.

51 Schrift-Proben aus der Buchdruckerey von Carl Dullo in Reval (Reval: Dullo, 1819), TLÜAR, IX-1437. 
Exemplare in ausländischen Bibliotheken, Archiven und Museen und deren Durchsicht de visu ist nicht immer möglich bzw. sehr schwierig. Die Digitalisierung alter Drucke kann dabei eine Hilfe sein, was europäische Bibliotheken und Archive entsprechend ihrer Möglichkeiten auch tun.

\section{ZUSAMMENFASSUNG}

Im Verlauf der Zusammenstellung der estnischen fremdsprachigen retrospektiven Bibliografie haben neben den bibliografischen Angaben die Verzierungselemente unsere Aufmerksamkeit gefesselt, weil sich die Vignetten in den Gratulations- und Funeraldrucken von den allgemeinen dekorativen Elementen unterscheiden. Jahrhunderte lang war der Tod für den Menschen fester Bestandteil des Lebens. Im Laufe der Zeit haben sich Begräbnisrituale herausgebildet, die abhängig von der sozialen Herkunft des Verstorbenen unterschiedlich durchgeführt wurden, doch das Ziel war es, die Erinnerung an den Verstorbenen durch Grabdenkmäler oder Funeraldrucke zu bewahren. Dies bestätigen die im 17. und 18. Jahrhundert gedruckten Leichenpredigten und Trauergedichtsammlungen. In den Drucken wurde der Tod durch verschiedene Symbole visualisiert, so auch durch die Vignetten, die unabhängig von Alter und Beruf verwendet werden konnten. In den in Estland erschienenen fremdsprachigen Drucken treten Totenschädel- und Sargmotive in den Vordergrund, etwas weniger wurde die Todessymbolik in Form von Sensenmann, Kreuz und Kruzifix verwendet.

Aufgrund der bis heute verzeichneten Angaben können wir sagen, dass Schmuckelemente in den Funeraldrucken den deutschen und den schwedischen Traditionen folgen, besonders in den Verzierungselementen des 17. Jahrhunderts ist die enge Verbindung zu der in schwedischen Buchdruckereien verwendeten Ornamentik zu sehen.
Tilu Reimo: Death! - Grave! - Decay! The Visualisation of Death in Foreign-Language Funeral Publications in 17th and 18Th CENTURY ESTONIA

Keywords: Funeral publications; 17th and 18Th Centuries; Symbols of Death; Vignettes

\section{SUMMARY}

The materials in the Retrospective National Bibliography of foreignlanguage publications printed in Estonia before 1830 provide various opportunities for analysing the production of local print shops. The article focuses on the illustrative elements in printed funeral sermons and works of poetry, which cast a light on the memorial and commemorative customs in the early modern period.

Visual decorative elements like headpieces and vignettes had a general symbolic meaning and were used to illustrate funeral texts irrespective of the age, vocation or position of the deceased. One objective was to remind the viewers of their own mortality. Skulls and coffins were among the main vignette motifs used to depict Death, and less often, Death was depicted as a skeleton or the Grim Reaper. Inscriptions added to the vignettes emphasised relevant passages from the Bible to strengthen one's faith. The fact that the same or similar vignettes were simultaneously used in different countries is noteworthy. The motifs for visualising Death and mourning used in the foreign-language funeral publications in Estonia are very similar to those used in Sweden and Finland during the same period.

\section{CV}

Tiiu Reimo (b. 1953), PhD, is book historian working as Editor-inChief of the Centre for Retrospective National Bibliography at the Academic Library of Tallinn University. She has worked for a long time at Baltica and the Rare Books Department of the Academic Library. From 2003 to 2013, she was Professor of Book Science at Tallinn University. She has published a monograph on the Tallinn book culture in the second half of the 18th century, and numerous articles on the history of books, printing and libraries in Estonia. 
